\title{
Interaction Mechanism and Coordinated Control of Commutation Failure Prevention in Multi-Infeed Ultra-HVDC System
}

\author{
Wei Yao $\mathbb{D}^{1},{ }^{1}$ Lingrao Wang $\mathbb{D}^{1},{ }^{1}$ Yongxin Xiong $\mathbb{D},{ }^{1}$ Yangchen Tan $\mathbb{D}^{2},{ }^{2}$ Dahu Li $\mathbb{D},{ }^{3}$ \\ Chenghao Li $(\mathbb{D})^{4}$ and Jinyu Wen $\left(\mathbb{D}^{1}\right.$ \\ ${ }^{1}$ State Key Laboratory of Advanced Electromagnetic Engineering and Technology, School of Electrical and Electronic Engineering, \\ Huazhong University of Science and Technology, Wuhan 430074, China \\ ${ }^{2}$ Northwest Branch of State Grid Corporation of China, Xian 710048, China \\ ${ }^{3}$ Smart Grid Hubei Electric Power Company Limited, Wuhan 430077, China \\ ${ }^{4}$ Electric Power Research Institute, State Grid Henan Electric Power Company, Zhengzhou 450052, China
}

Correspondence should be addressed to Wei Yao; w.yao@hust.edu.cn

Received 9 October 2021; Revised 15 January 2022; Accepted 17 January 2022; Published 27 February 2022

Academic Editor: Gulshan Sharma

Copyright (c) 2022 Wei Yao et al. This is an open access article distributed under the Creative Commons Attribution License, which permits unrestricted use, distribution, and reproduction in any medium, provided the original work is properly cited.

\begin{abstract}
Commutation failure (CF) is an inherent drawback of line-commutated converter high-voltage DC (LCC-HVDC) system. And CF prevention (CFPREV) control is widely applied in UHVDC lines to mitigate the subsequent CF. However, the formation of a multi-infeed UHVDC system induces power exchange and voltage interaction between AC buses during the transient process, making the behaviors and mitigation of CFs more complex. Consequently, exploring the impact of CFPREV control on adjacent stations and optimizing the CFPREV control to adapt to the interaction characteristics in a multi-infeed system is necessary. Considering the interaction characteristics, the impact of CFPREV control on the commutation process of inverters in a multiinfeed system is analyzed through formula derivation and simulation verification. It is found that although CFPREV control can effectively mitigate the CFs of the inverter near the fault, it will further increase the risk of concurrent CF (CCF) of remote inverters due to the interaction on the AC side. To solve this problem, a coordinated control scheme of CFPREV controls in a multi-infeed UHVDC system is proposed. The output of the CFPREV control of the inverter near the fault can be adjusted adaptively according to the commutation margins of remote inverters. And the probability of CCF caused by CFPREV control is consequently decreased. Case studies are conducted based on Henan provincial multi-infeed UHVDC system in China to verify the analysis result and the effectiveness of the coordinated control scheme of CFPREV controls.
\end{abstract}

\section{Introduction}

Ultra-HVDC (UHVDC) transmission technology has been extensively utilized around the world due to its well-known advantage of long-distance bulk power transmission and lower operating power losses [1]. And commutation failure (CF) is a frequent and adverse event in LCC-HVDC systems, usually occurring as the consequence of the short-circuit fault in the AC system on the inverter side [2, 3]. Statistics show that a total number of $60 \mathrm{CFs}$ were recorded in East China Power Grid from 2011 to 2018, and up to four HVDC lines suffered from a temporary interruption of the power transmission and misoperation of the protective relays in the severest cases $[4,5]$. A CF prevention (CFPREV) control scheme was firstly introduced by [6] in 2002 to mitigate the subsequent CF in a single HVDC line, and it has been widely applied in UHVDC transmission projects at present as the main control strategy for CF mitigation.

As the continuous construction of UHVDC links $[7,8]$, situations are arising that two or more UHVDC converters terminating into a regional AC system are located electrically in close proximity. And such system is termed as "multiinfeed UHVDC system," which has been formed in the South China Power Grid and the East China Power Grid [9]. 
Multi-infeed UHVDC system induces the formation of electrical interaction channels between inverter stations through AC network, and there will be power exchange and voltage interaction between $\mathrm{AC}$ buses during the transient process. Therefore, the impacts of CF and CFPREV control on the operation characteristics of one inverter station will affect the operation parameters of adjacent stations through AC side interaction, making the behavior of CF and its mitigation in a multi-infeed system more complex [10]. Reference [11] defines the CFs in multi-infeed UHVDC systems into two types, local CF (LCF) and concurrent CF (CCF). The former refers that a fault applied near a given converter bus causes the converter to suffer $\mathrm{CF}$, while the latter refers to the fact that a fault close to a converter causes CF at the local as well as the adjacent converters. In 2011, CCFs occurred at four inverter stations due to AC faults in the East China Power Grid. And only two months after this accident, another disturbance in the AC system leads to three inverter stations in the East China Power Grid confronting CCFs, and UHVDC lines are forced blocking. Accordingly, the mitigation of CFs in multiinfeed UHVDC systems is an important topic in power grid planning and operation.

The interaction mechanism analysis in the multi-infeed system has been investigated sufficiently in recent years [12-14]. The article [15] comes up with AC/DC voltage interaction factor (ADVIF) to assess voltage interaction between AC buses through impedance parameters of AC network. References $[16,17]$ consider the distribution of real and reactive power in interaction quantization, using the minimum eigenvalue of AC-DC Jacobian matrix and interactive effective short-circuit ratio, respectively, to determine the stability condition of the multi-infeed system. References $[18,19]$ point out that the harmonic component generated in the transient process is also involved in the interaction between inverter stations and accordingly put forward critical indexes to evaluate the risk of CCFs caused by interaction. Although elements including AC network impedance, power distribution, and harmonic components have been discussed, none of the above researches take the impact of DC control strategy on the interaction between stations into consideration. Particularly, CFPREV control is the DC control strategy mostly applied in each UHVDC line. And it is clearly claimed in [20] that the firing angle alteration of CFPREV control will change the reactive power absorption of the inverter station and therefore influence the voltage of the AC bus. So the exploration of the impact of CFPREV control on the commutation process in adjacent stations through $\mathrm{AC}$ side interaction in multi-infeed UHVDC system is necessary and still lacking.

In addition to the $\mathrm{CF}$ mechanism analysis, various $\mathrm{CF}$ mitigation methods have been developed. At present, the commonly used strategies mainly fall into the following categories: (1) modifying the layout, capacity allocation, and switching control of reactive power compensation devices [21-24], (2) reforming the converter topology [25], and (3) optimizing the DC control scheme [6, 10, 26, 27]. Reactive power compensation equipment is not only expensive but also increases short-circuit current of the AC system. The modification of the converter structure includes the improvement of thyristor performance and adding capacitors, inductors, and thyristors to the converter topology to reduce the risk of CF. However, due to voltage grade, transmission capacity, technical limitation, and other factors, its engineering feasibility remains to be further verified. Compared with the first two categories, the optimization of DC control strategy has more advantages in economy and feasibility, owning more general application in engineering for $\mathrm{CF}$ mitigation. The main DC control schemes to mitigate $\mathrm{CF}$ including voltage dependent current order limitation (VDCOL) control and CFPREV control, and the latter will be studied as the focus of this paper. The mechanism of CFPREV can be simply summarized as follows: (1) detecting the faults in the AC system through zero-sequence detection and abc- $\alpha \beta$ transformation, (2) determining whether the AC voltage disturbance exceeds the threshold, and (3) advancing the firing angle if the fault is severe enough. Although CFPREV control is effective in mitigating the multivalve and repeated CFs, it is originally designed for the single-infeed system twenty years ago. As the regional multi-infeed UHVDC system has been widely formed in recent years, the CFPREV control should also be optimized accordingly.

To cope with the two research tasks introduced above, this paper investigates the impact of CFPREV control on the commutation processes in multi-infeed UHVDC systems considering the interaction between adjacent inverter stations. Analysis results show that when an AC fault occurs near an inverter station, its associated CFPREV control will alter the firing angle rapidly to mitigate the LCF. Nevertheless, the mechanism of CFPREV control determines that it will also increase the probability of CCFs in remote stations due to the reactive power exchange and voltage interaction on the AC side. To address this problem, a coordinated control scheme of CFPREV controls in a multiinfeed UHVDC system is proposed to prevent the CCFs. This coordinated controller can adaptively adjust the output of CFPREV control according to the extinction angle drop of the inverters in other UHVDC lines, so as to reduce the adverse effect on the commutation processes in remote inverter stations. Simulation results of the Henan provincial multi-infeed UHVDC system in China verify the effectiveness of the coordinated control scheme under reasonable transmission delay and noise interference situations.

The main contributions of this paper are listed as follows:

(i) The influence of CFPREV control on the commutation processes considering interaction in the multi-infeed UHVDC system is deduced. Analysis results show that when CFPREV control mitigates the LCF in its corresponding inverter station, it will also increase the probability of CCFs in adjacent inverter stations.

(ii) A coordinated control scheme of CFPREV controls is proposed for multi-infeed UHVDC systems to mitigate the adverse impact of the CFPREV control on adjacent UHVDC lines. The coordinated controller monitors the extinction angle of adjacent UHVDC lines and adjusts its output adaptively.

(iii) Simulation results of the practical multi-infeed UHVDC project show that the coordinated control 
scheme can reduce the probability of the CCFs in adjacent UHVDC lines caused by the CFPREV control effectively. And the sensitivity of the scheme to transmission delay and noise interference in engineering practice is also discussed.

The rest of this paper is organized as follows. Section 2 analyzes the mechanism of CFPREV control and its impact on the commutation process of the inverters in multi-infeed systems. Section 3 proposed a coordinated control scheme of CFPREV controls for the multi-infeed UHVDC system. Case studies are undertaken on the Henan provincial multiinfeed UHVDC system of China to verify the effectiveness of the coordinated control scheme of CFPREVs under transmission delay consideration in Section 4. Finally, conclusions are drawn in Section 5.

\section{Influence of CFPREV on Multi-Infeed System}

2.1. Multi-Infeed UHVDC System. The diagram of the Henan provincial multi-infeed UHVDC system consisting of two UHVDC transmission lines and three inverter stations on the receiving end is shown in Figure 1. UHVDC system 1 is a regular UHVDC transmission line, whose inverters terminate at $\mathrm{AC}$ bus 1 of $500 \mathrm{kV}$. However, UHVDC system 2 is a UHVDC transmission line with a hierarchical connection, where high and low side inverters terminate to two adjacent convert buses. Specifically, the two twelve-pulse inverters I and IV connected directly with $\pm 800 \mathrm{kV}$ DC lines are called high side inverters, terminating at $\mathrm{AC}$ bus 2 . And the two twelve-pulse inverters II and III connected directly with the neutral point are named as low side inverters, terminating at AC bus 3. Such connection mode divides UHVDC system 2 into two parts with a half capacity to release the stress of voltage support capacity of AC systems connected with convert buses. Note that the research in this model is concentrated on the DC transmission part, so the entire AC network connected with each convert bus is equivalent to an electromotive force $E_{i}$ and system impedance $Z_{i}$ by Thevenin theorem. And $U_{i} \angle \varphi_{i}$ is the voltage of bus $i ; Z_{i j} \angle \theta_{i j}$ is the impedance of the tie line between bus $i$ and $j$. The impedance parameters of the receiving end network are shown in Table 1.

\subsection{Impact of CFPREV on Local Commutation Failure}

2.2.1. Commutation Failure Mechanism. The equivalent circuit of a 6-pulse Graetz bridge inverter connected to the $\mathrm{Y}-\mathrm{Y}$ transformer is shown in Figure 2. Under stable operation, the six thyristor valves named VT1 to VT6 are fired in sequence, and the conduction interval of the adjacent valve is $(3 / \pi)$. The firing angle is denoted as $\alpha$, and the leading firing angle $\beta=\pi-\alpha . U_{a}, U_{b}$, and $U_{c}$ are the three-phase voltage at the inverter side, $L_{c}$ is the commutation inductance, and $I_{d}$ and $u_{d}$ are DC voltage and current, respectively. Due to the leakage inductance of the converter transformer and the impedance of the AC system, the transfer of the current from one valve to another requires a certain time, which is termed as commutation time or overlap angle, denoted as $\mu$. Also, after the valve current goes to zero, the thyristor still needs to withstand the reverse voltage for a certain time to establish a forward voltage blocking capability. Consequently, the interval between the instant when the valve current goes to zero to the time that the commutation voltage is zero is defined as deionisation time or extinction angle, denoted as $\gamma$. If the deionisation time is insufficient, and $\gamma<\gamma_{\min }$, this thyristor will regain current, and CF occurs [28].

Voltage-time area (VTA) theory is widely used in the mechanism analysis of CF, and it can be expressed as follows. Taking the commutation process from VT3 to VT5 in an inverter connected to a Y-Y transformer as an example, the diagram of the commutation process and the equivalent circuit is shown in Figure 3. This process can be divided into three stages: (1) before $t_{1}$ : the commutation process has not begun, VT4 and VT3 turn on and constitute a circuit; (2) $t_{1}-t_{2}$ : the commutation from VT3 to VT5 is in process. There are three thyristors (VT4, VT3, and VT5) turning on during this period. According to Figure 3(b), the current in VT3 is transferring to VT5, so $i_{3}$ keeps decreasing and $i_{5}$ rises from 0 ; (3) after $t_{2}$ : the commutation from VT3 to VT5 is finished, $i_{3}$ drops to zero, and VT3 turns off. VT4 and VT5 turn on and constitute a circuit. The relationship between the commutation voltage $U_{\mathrm{cb}}$ and the valve current $i_{3}, i_{3}$ and $i_{5}$ can be described as

$$
U_{c}-U_{b}=L_{c} \frac{\mathrm{d} i_{5}}{\mathrm{~d} t}-L_{c} \frac{\mathrm{d} i_{3}}{\mathrm{~d} t}
$$

$I_{d}$ is constant during the normal commutation process, whereas its distribution in VT3 and VT5 changes:

$$
i_{5}=I_{d}-i_{3} \text {. }
$$

Substitute (2) to (1) and integrate both sides of (1) from $t_{1}$ to $t_{2}$ to obtain the following equation:

$$
\int_{t_{1}}^{t_{2}}\left(U_{c}-U_{b}\right) \mathrm{d} t=\int_{t_{1}}^{t_{2}} L_{c}\left(\mathrm{~d} I_{d}-2 \mathrm{~d} i_{3}\right),
$$

where $t_{1}$ is the firing time when commutation begins, corresponding to firing angle $\alpha$, and $t_{2}$ is the time when the current in VT3 drops to zero and commutation finishes, corresponding to extinction angle $\gamma$. The period between $t_{1}$ and $t_{2}$ is commutation process, denoted as commutation overlap $\mu$. When the commutation process ends, $i_{5}\left(t_{2}\right)=I_{d}\left(t_{2}\right)$, and $i_{3}\left(t_{2}\right)=0$, then (3) can be written as

$$
\int_{t_{1}}^{t_{2}}\left(U_{c}-U_{b}\right) \mathrm{d} t=L_{c}\left(I_{d}\left(t_{1}\right)+I_{d}\left(t_{2}\right)\right) .
$$

The left side of (4) is the VTA demand for a normal commutation, and the right side of (4) is the VTA provided by the system. Consequently, only when (5) is satisfied can the commutation process be successful.

$$
L_{c} I_{d}\left(t_{2}\right)+L_{c} I_{d}\left(t_{1}\right) \leq \int_{t_{1}}^{t_{2 \max }}\left(U_{c}-U_{b}\right) \mathrm{d} t
$$

The left side of (5) is denoted as $S_{\text {demand }}$, which is determined by $I_{d}$ when $L_{c}$ keeps constant in AC fault transient process. The right side of (5) is the maximum voltage-time area that can be provided by the system in a critical situation 


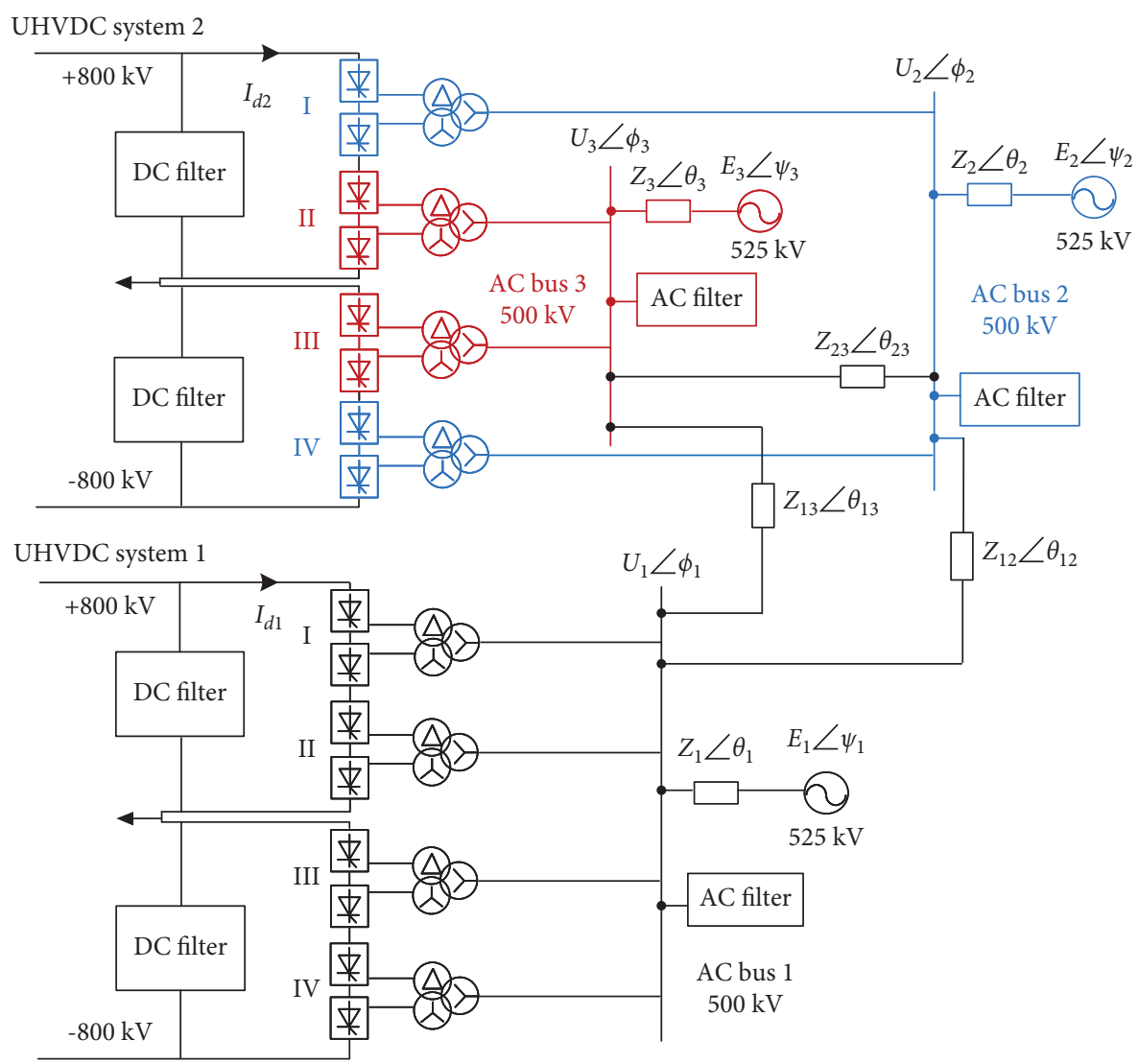

FIgURE 1: Structure of Henan provincial multi-infeed UHVDC system in China.

TABLE 1: Parameters of the equivalent model of Henan provincial multi-infeed UHVDC system.

\begin{tabular}{lccc}
\hline Parameter & Value $/ \Omega$ & Parameter & Value $(\mathrm{L}-\mathrm{L}) / \mathrm{kV}$ \\
\hline$Z_{1} \angle \theta_{1}$ & $7.389 \angle 85^{\circ}$ & $E_{1} \angle \psi_{1}$ & $725 \angle 0^{\circ}$ \\
$Z_{2} \angle \theta_{2}$ & $24.037 \angle 85^{\circ}$ & $E_{2} \angle \psi_{2}$ & $770 \angle 0^{\circ}$ \\
$Z_{3} \angle \theta_{3}$ & $25.035 \angle 85^{\circ}$ & $E_{3} \angle \psi_{3}$ & $770 \angle 0^{\circ}$ \\
$Z_{12} \angle \theta_{12}$ & $65.847 \angle 85^{\circ}$ & $U_{1} \angle \varphi_{1}$ & $743 \angle 12^{\circ}$ \\
$Z_{13} \angle \theta_{13}$ & $84.121 \angle 85^{\circ}$ & $U_{2} \angle \varphi_{2}$ & $744 \angle 15^{\circ}$ \\
$Z_{23} \angle \theta_{23}$ & $16.786 \angle 85^{\circ}$ & $U_{3} \angle \varphi_{3}$ & $744 \angle 15^{\circ}$ \\
\hline
\end{tabular}

that $\gamma=\gamma_{\min }$, denoted as $S_{\max }$, as the shaded area shown in Figure 3(b), mainly determined by the integral time and commutation voltage. As a result, the arising DC current, the commutation voltage magnitude reduction, and zerocrossing phase shift caused by voltage distortion can all be the influential factors of CF.

2.2.2. Mechanism of CFPREV. According to the analysis above, the mechanism of LCF under inverter side AC fault can be concluded concisely as follows. After the short-circuit fault occurs in receiving system, the voltage dip of the $\mathrm{AC}$ bus in the inverter station causes the DC current to rise, and $S_{\text {demand }}$ is increasing consequently. However, the commutation voltage magnitude reduction and zero-crossing phase shift make the $S_{\max }$ decrease and gradually fail to provide sufficient extinction angle. When $S_{\max }<S_{\text {demand }}$, the CF occurs. To suppress the CF, one idea is lengthening the integral time by advancing the firing instant to enlarge $S_{\max }$. And CFPREV control based on fast AC fault detection and advancing firing angle control is originally introduced in [6] in 2002. CFPREV control is has been widely applied in UHVDC transmission projects in China due to its remarkable effectiveness in mitigating the multi-valve and repeated CFs.

The control diagram of CFPREV is shown in Figure 4. $\alpha$ (deg) is the firing angle calculated by inverter control. The mechanism of CFPREV is to provide a firing angle alteration order $\Delta \alpha_{\mathrm{CFP}}$ (deg) by detecting the AC bus voltage dip caused by AC faults and then make $\Delta \alpha_{\mathrm{CFP}}$ difference with $\alpha$ to acquire a further decreased firing order $\alpha_{\text {ord }}$ (deg). The AC fault detection consists of two parallel parts: zero-sequence detection for asymmetric fault detection and abc- $\alpha \beta$ transformation for symmetric fault detection. The input real-time measured parameters $\dot{U}_{a}, \dot{U}_{b}$, and $\dot{U}_{c}$ are perunited values of the instantaneous three-phase voltage of receiving AC bus. The zero-sequence of voltages can be calculated as

$$
\left|3 \dot{U}_{0}\right|=\left|\dot{U}_{a}+\dot{U}_{b}+\dot{U}_{c}\right|
$$

Three-phase voltages are symmetrical in steady-state operation, and the value of $\left|3 \dot{U}_{0}\right|$ should be zero. While in asymmetric AC fault situation, the value of $\left|3 \dot{U}_{0}\right|$ will rise up. When $\left|3 \dot{U}_{0}\right|$ exceeds the threshold $U_{0 \_ \text {th }}$, the enable signal of asymmetric fault control $S_{0}$ will step up from 0 to 1 . Otherwise, $S_{0}$ should keep as 0 , and this part remains 


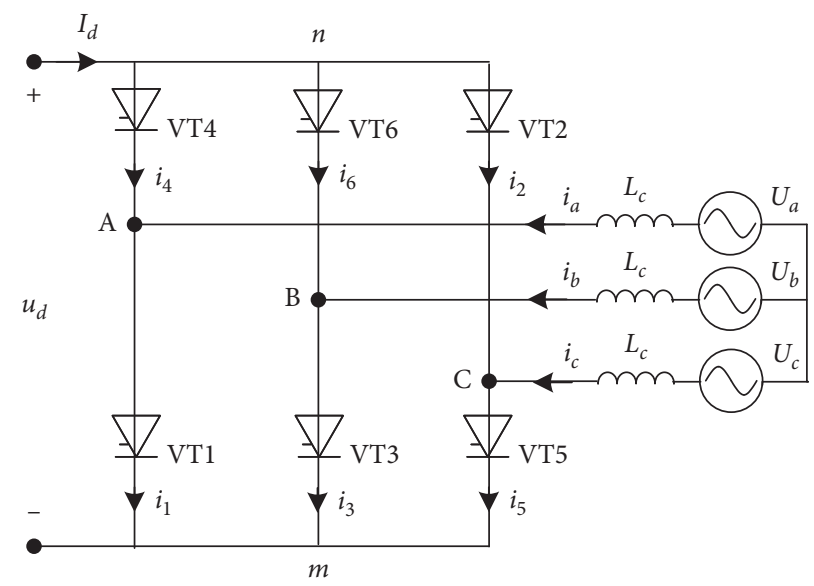

Figure 2: Equivalent circuit of a 6-pulse Graetz bridge inverter.

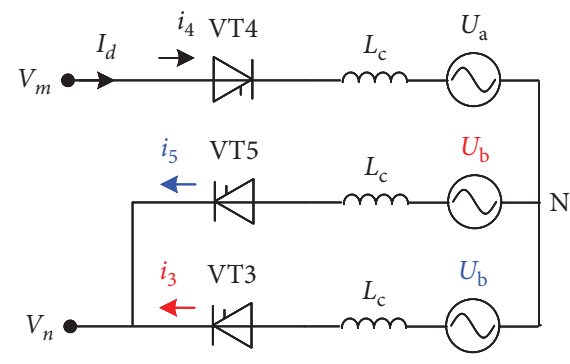

(a)

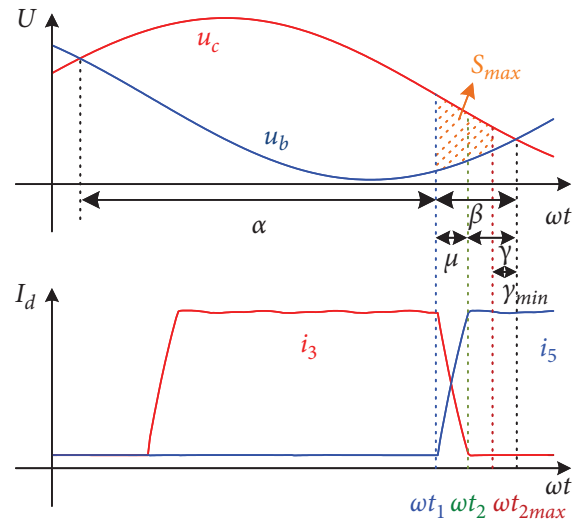

(b)

FIgURE 3: Equivalent circuit and diagram of commutation from VT3 to VT5. (a) Equivalent circuit of commutation. (b) Diagram of commutation process.

uninitialized. Because $S_{0}$ usually fluctuates between 0 and 1 during the fault transient process, it needs to be modified by a monostable block with $20 \mathrm{~ms}$ pulse duration to acquire a stable control enable signal. As the output of the monostable block is 1 , the value of $\left|3 \dot{U}_{0}\right|$ will be multiplied by a coefficient $k_{\mathrm{CFP}}$ to acquire a leading firing angle adjustment value $\Delta \beta_{0}$, which will be sent to the Max Choice block and remains to be output.

The mechanism of abc- $\alpha \beta$ transformation part is to use one rotating vector in the $\alpha \beta$-plane to comprehensively represent three-phase voltages and then judge the symmetric fault by the magnitude of the rotating vector. Actually, such an algorithm is available under both symmetric and asymmetric faults detection:

$$
\left\{\begin{array}{c}
U_{\alpha}=\frac{2}{3} \dot{U}_{a}-\frac{1}{3}\left(\dot{U}_{b}+\dot{U}_{c}\right), \\
U_{\beta}=\frac{\sqrt{3}}{3}\left(\dot{U}_{b}-\dot{U}_{c}\right), \\
U_{\alpha \beta}=\sqrt{U_{\alpha}^{2}+U_{\beta}^{2}},
\end{array}\right.
$$

$$
U_{\alpha \beta}=\sqrt{\frac{2\left[\left(\dot{U}_{a}-\dot{U}_{b}\right)^{2}+\left(\dot{U}_{b}-\dot{U}_{c}\right)^{2}+\left(\dot{U}_{c}-\dot{U}_{a}\right)^{2}\right]}{9}} .
$$

According to (9), the value of $U_{\alpha \beta}$ is the amplitude of AC voltage 1 (p.u). While in short-circuit AC fault cases, the fault component $\left(1-U_{\alpha \beta}\right)$ comprehensively represents the sag of three-phase voltage. When $\left(1-U_{\alpha \beta}\right)$ exceeds the threshold $U_{\alpha \beta_{-} \text {th }}$, the enable signal of this block $S_{\alpha \beta}$ will step up from 0 to 1 . Otherwise, $S_{\alpha \beta}$ should keep as 0 , and this part remains uninitialized. Similarly, $S_{\alpha \beta}$ needs to be modified by a monostable block with $20 \mathrm{~ms}$ pulse duration to acquire a stable control enable signal. As the output of the monostable block is 1 , the value of $\left(1-U_{\alpha \beta}\right)$ will be multiplied by a coefficient $k_{\mathrm{CFP}}$ to acquire a leading firing angle adjustment value $\Delta \beta_{\alpha \beta}$, which will be sent to the Max Choice block and remains to be output.

According to (9), the amplitude of $U_{\alpha \beta}$ is only calculated from the positive and negative sequence components of $\mathrm{AC}$ voltage, excluding the zero-sequence component. Therefore, in CFPREV control, abc- $\alpha \beta$ transformation and zero-sequence detection are calculated in parallel to effectively 


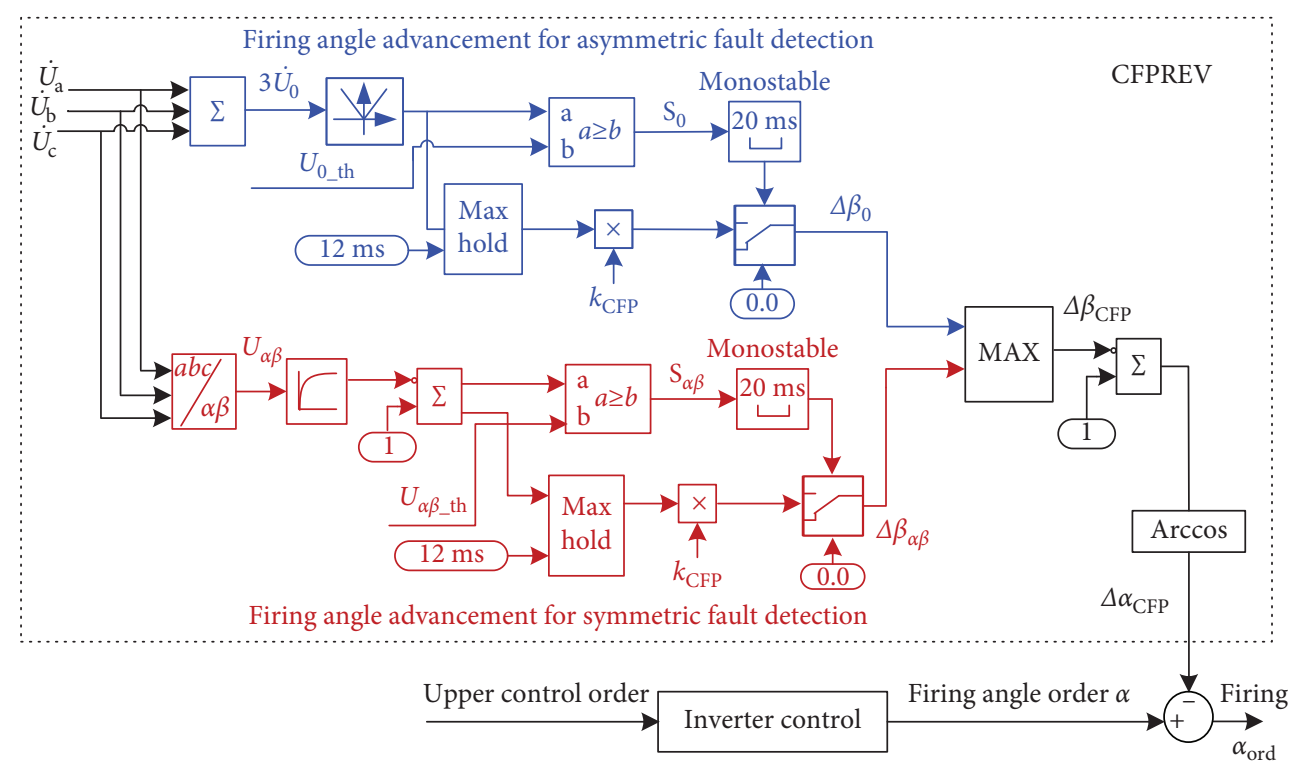

Figure 4: Block diagram of CFPREV.

cover the rapid detection of all kinds of $\mathrm{AC}$ short-circuit faults. In asymmetric fault case where abc- $\alpha \beta$ transformation and zero-sequence detection are activated at the same time, the maximum value of $\Delta \beta_{\alpha \beta}$ and $\Delta \beta_{0}$ will be chosen as $\Delta \beta_{\mathrm{CFP}}$.

\subsubsection{Impact of CFPREV on Commutation Process in the} Inverter Station near the Fault. The working mechanism of CFPREV which is advancing the firing instant will cause inverters to absorb more reactive power and eventually lead to a further descent of the voltage amplitude of the converter bus $[29,30]$. The impact of the firing angle alteration of CFPREV control on the reactive power consumption of inverters can be deduced as follows [31]. The valve current on the DC side and DC voltage can be described as

$$
\begin{gathered}
I_{\mathrm{d}}=\frac{\sqrt{2} U_{\mathrm{LL}}}{2 \omega L_{\mathrm{c}}}(\cos \alpha+\cos \gamma), \\
\Delta U_{c}=R_{c} I_{d}=\frac{3}{\pi} \omega L_{c} I_{d} \\
U_{d}=\frac{3 \sqrt{2}}{\pi} U_{\mathrm{LL}} \cos \gamma-\Delta U_{\mathrm{c}}=\frac{3 \sqrt{2} U_{\mathrm{LL}}}{2 \pi}(\cos \gamma-\cos \alpha),
\end{gathered}
$$

where $U_{\mathrm{LL}}$ is the RMS value of line-to-line AC voltage. $\Delta U_{\mathrm{c}}$ refers to the influence of commutation overlap $\mu$ on $U_{d}$, and $R_{c}$ is the equivalent commutation impedance on inverter side. Assuming that there are no active power losses during the inverter,

$$
U_{d} I_{d}=\sqrt{3} U_{\mathrm{LL}} I_{d} \cos \varphi,
$$

where $I_{L}$ is the RMS value of the fundamental component of AC current, and $\varphi$ is the phase between voltage and current. According to Fourier decomposition, the relationship between $I_{L}$ and $I_{d}$ can be described as

$$
I_{L}=\frac{1}{\sqrt{2} \pi} \int_{-\pi}^{\pi} I_{d} \cos \omega t \mathrm{~d} \omega t=\frac{\sqrt{6}}{\pi} I_{d}
$$

Combining (10)-(14),

$$
\cos \varphi=\frac{1}{2}(\cos \gamma-\cos \alpha)
$$

where $\alpha \in\left[90^{\circ}, 180^{\circ}\right], \gamma=\pi-\alpha-\mu$, so the reactive power consumption of DC system is

$$
Q_{d}=\sqrt{3} U_{\mathrm{LL}} I_{L} \sin \varphi=\sqrt{3} U_{\mathrm{LL}} I_{L} \sqrt{1-\frac{(\cos \gamma-\cos \alpha)^{2}}{4}}
$$

According to (16), $Q_{d}$ increases as the reduction of $\alpha$ and the rise of $\gamma$, which is exactly consistent with the mechanism of CFPREV control. So, the application of CFPREV control will enlarge the commutation margin of inverters, augmenting the reactive power consumption of the DC system, and further cause the voltage drop of the AC bus in the fault transient process. The theoretical analysis above is verified by the simulation in the Henan provincial multi-infeed UHVDC system shown in Figure 1 in PSCAD/EMTDC. For simplification, the inverter group terminating at $\mathrm{AC}$ bus 1 through transformers is named as inverter 1. A three-phase-to-ground fault with the inductance of $0.1 \mathrm{H}$ is set near AC bus 1 . The fault starts at $1 \mathrm{~s}$ and lasts for $0.1 \mathrm{~s}$. CFPREV control is applied in inverter 1 , and the values of $k_{\mathrm{CFP}}$ are set as 0 (no CFPREV control), 0.02, and 0.06, respectively. The impact of CFPREV control with various $k_{\text {CFP }}$ values on inverter station 1 is compared in Figure 5. It has been interpreted that $k_{\mathrm{CFP}}$ is the coefficient between the abnormal component and the firing angle advancement value. According to Figure 5(a), as the $k_{\mathrm{CFP}}$ increases from 0 to $0.06, \Delta \alpha_{\mathrm{CFP}}$ goes up consistently. Although a larger $\Delta \alpha_{\text {CFP }}$ could provide more sufficient $S_{\max }$ in Figure 5(d), which greatly reduces the probability of LCF, it will also cause more reactive power consumption of the inverter 1 


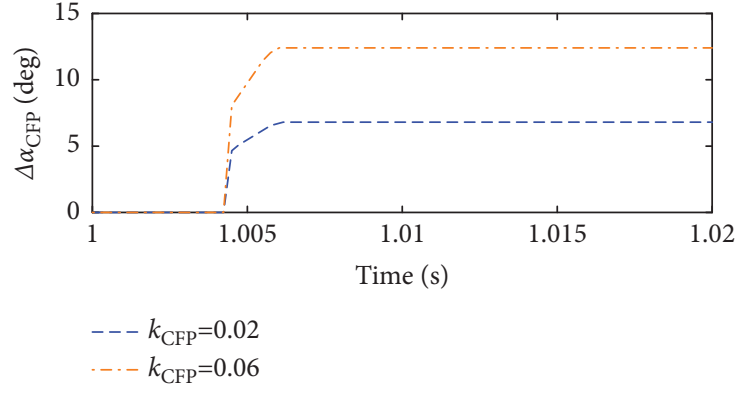

(a)

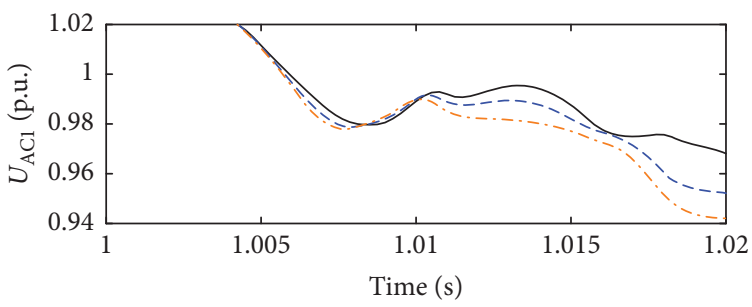

$$
\begin{aligned}
& -k_{\mathrm{CFP}}=0 \\
& ---k_{\mathrm{CFP}}=0.02 \\
& --k_{\mathrm{CFP}}=0.06
\end{aligned}
$$

(c)

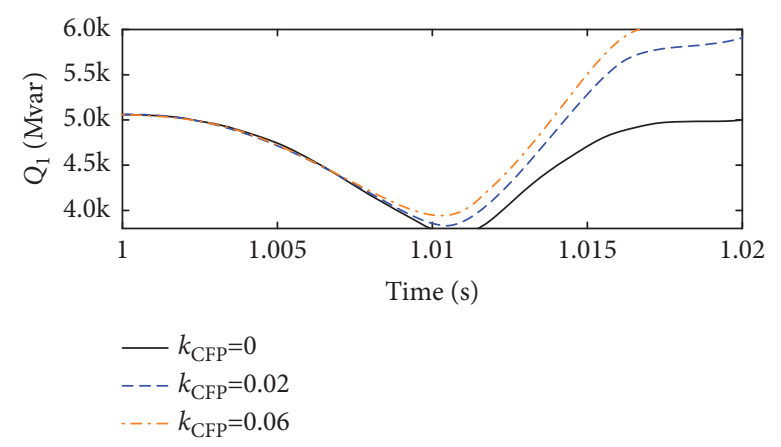

(b)

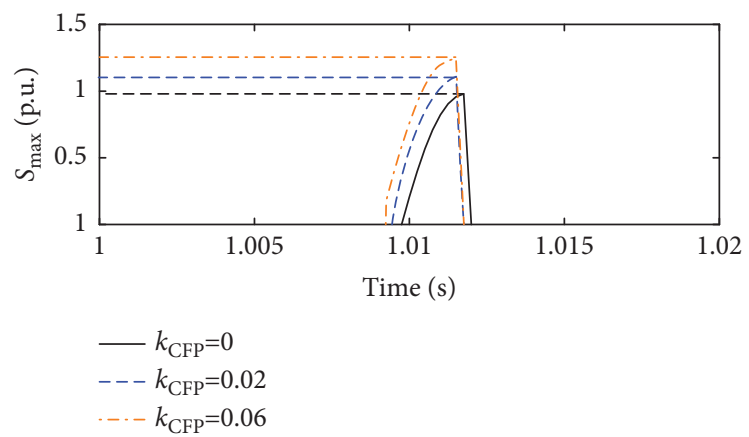

(d)

FIGURE 5: The impact of the CFPREV strategy on the inverter station near the fault. (a) $\alpha$ advancement order of CFPREV control; (b) reactive power absorbed by inverter 1; (c) RMS voltage of AC bus 1; (d) $S_{\max }$ of AC bus 1.

in Figure 5(b), resulting in a larger voltage drop of AC bus 1 in Figure 5(c). In addition to the three-phase-to-ground fault case shown in Figure 5, the parameters of inverter station 1 at $1.02 \mathrm{~s}$ under other types of fault cases are summarized in Table 2 . The AC system faults with the inductance of $0.1 \mathrm{H}$ in Table 2 all occur at $1 \mathrm{~s}$ and last for $0.1 \mathrm{~s}$. The results of all types of fault in Table 2 are consistent with those in Figure 5 and (15); that is, the increase of coefficient $k_{\mathrm{CFP}}$ not only makes CFPREV controller output higher $\Delta \alpha_{\mathrm{CFP}}$ value and provides more commutation margin $S_{\max }$ in fault transient process. It but also causes higher reactive power consumption of the inverter and more voltage reduction of $\mathrm{AC}$ bus.

A more direct comparison of the LCF mitigation effect of CFPREV control with various values of $k_{\mathrm{CFP}}$ is represented in Figure 6, where the fault set near AC bus 1 lasts for $0.1 \mathrm{~s}$. The fault inductance is set from 0 to $0.15 \mathrm{H}$, and the starting time of fault is set from $1 \mathrm{~s}$ to $1.009 \mathrm{~s}$. The blue boxes in this figure reveal that in some serious cases where the fault inductance is less than $0.1 \mathrm{H}, k_{\mathrm{CFP}}$ of 0.06 tends to have a better mitigation effect on LCFs. And there are no red boxes in Figure 6, meaning that within the range of $0.02-0.06$, a larger $k_{\mathrm{CFP}}$ will not result in a worse effect on LCFs mitigation.

\subsection{Impact of CFPREV on Commutation Process in Remote} Inverter Stations. According to the analysis in the above section, within a certain range, a larger value of $k_{\text {CFP }}$ will have a better performance on mitigating LCFs, and it will also put forward a higher reactive power requirement of its corresponding DC systems. In a multi-infeed system, the inverter stations have electrical interaction through the AC network. The higher reactive power consumption in the inverter station near the fault will force more reactive power flows out of remote stations through tie lines for voltage support, which will cause further voltage reductions of the remote AC buses. The extinction angle of the inverter connected with bus $i$ in a multi-infeed system under steady can be deduced as follows:

$$
\gamma_{i}=\arccos \left(\frac{\sqrt{2} n_{i} I_{d i} X_{c i}}{V_{i}}-\cos \alpha_{i}\right) .
$$

Assume that the voltage reduction of bus $i$ in disturbance is $\Delta V_{i}$; then, the extinction angle is

$$
\gamma_{i}=\arccos \left(\frac{\sqrt{2} n_{i} I_{d i} X_{c i}}{V_{i 0}-\Delta V_{i}}-\cos \alpha_{i}\right) .
$$

According to (18), the voltage reduction caused by the reactive power exchange between inverter stations will decrease the extinction angle of remote inverter stations, consequently leading to a worse commutation condition and even CF under serious circumstances.

The theoretical analysis above is also verified by the simulation results following. The high side inverter group terminating at AC bus 2 through transformers is named as inverter 2, and the low side inverter group terminating at AC bus 3 is named inverter as 3. When a three-phase-to-ground fault is set near AC bus 1, the impacts of CFPREV control of inverter 1 with $k_{\mathrm{CFP}}$ at 0 (no CFPREV control), 0.02 , and 0.06 on the commutation process of inverter 2 are illustrated in 
TABLE 2: Impact of $k_{\text {CFP }}$ values on its associated inverter station.

\begin{tabular}{|c|c|c|c|c|c|}
\hline \multirow{2}{*}{ Fault type } & \multirow{2}{*}{$k_{\mathrm{CFP}}$} & \multicolumn{4}{|c|}{ Parameters of inverter station $1(t=1.02 \mathrm{~s})$} \\
\hline & & $\Delta \alpha_{\mathrm{CFP} 1}(\mathrm{deg})$ & $Q_{1}(\mathrm{M}$ var $)(\mathrm{k})$ & $U_{\mathrm{AC} 1}$ (p.u.) & $S_{\max 1}$ (p.u.) \\
\hline \multirow{3}{*}{ LLLG fault } & 0 & 0.0 & 5.5 & 0.968 & 0.99 \\
\hline & 0.02 & 7.0 & 5.9 & 0.953 & 1.08 \\
\hline & 0.06 & 13.5 & 6.4 & 0.941 & 1.27 \\
\hline \multirow{3}{*}{ LLG fault } & 0 & 0 & 4.4 & 0.962 & 0.97 \\
\hline & 0.02 & 6.8 & 5.7 & 0.941 & 1.05 \\
\hline & 0.06 & 14.2 & 6.1 & 0.928 & 1.33 \\
\hline \multirow{3}{*}{ LL fault } & 0 & 0 & 4.5 & 0.983 & 1.10 \\
\hline & 0.02 & 6.6 & 5.4 & 0.976 & 1.24 \\
\hline & 0.06 & 12.2 & 6.0 & 0.962 & 1.31 \\
\hline \multirow{3}{*}{ SLG fault } & 0 & 0 & 5.0 & 0.998 & 1.19 \\
\hline & 0.02 & 5.4 & 5.7 & 0.995 & 1.31 \\
\hline & 0.06 & 10.7 & 6.0 & 0.988 & 1.37 \\
\hline
\end{tabular}

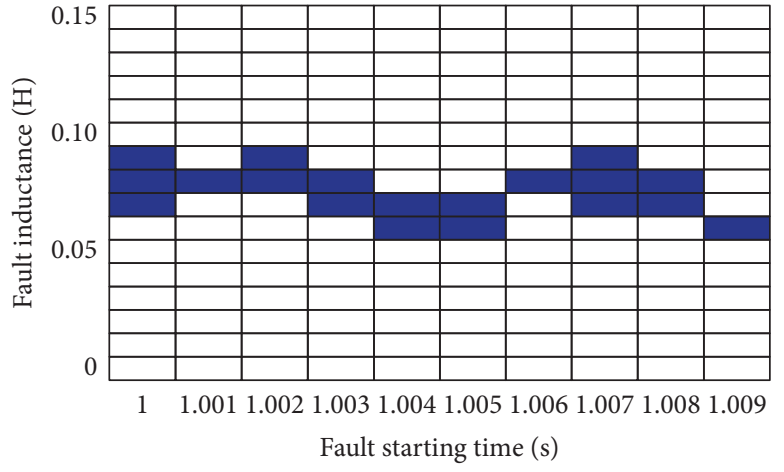

$\square$ Suppression effects with $k_{\mathrm{CFP}}$ at 0.06 and 0.02 are same

$\square$ Suppression effects with $k_{\mathrm{CFP}}$ at 0.06 is better than that at 0.02

Suppression effects with $k_{\mathrm{CFP}}$ at 0.06 is worse than that at 0.02

(a)

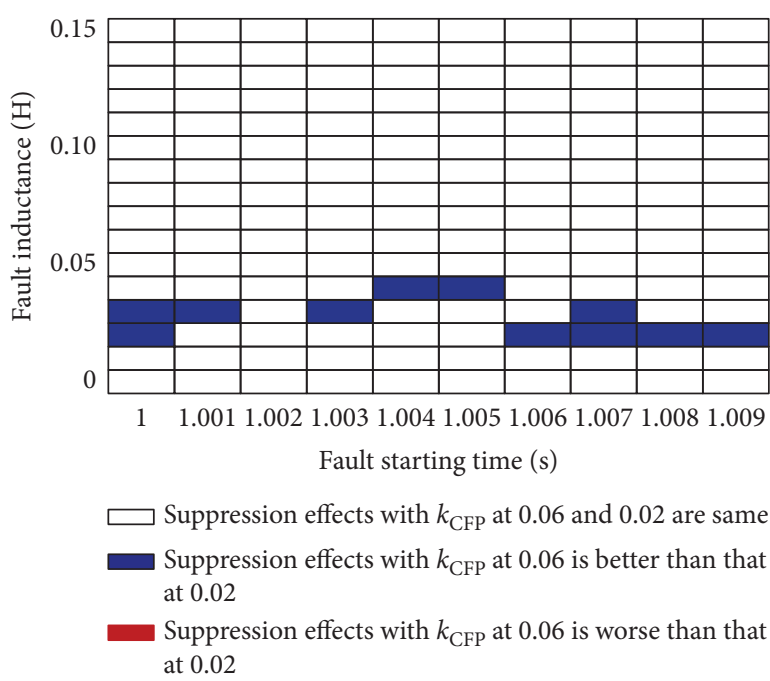

(c)

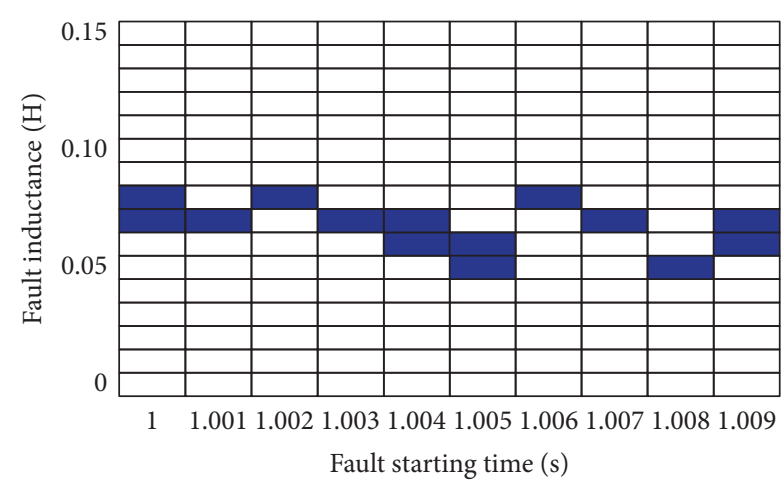

$\square$ Suppression effects with $k_{\mathrm{CFP}}$ at 0.06 and 0.02 are same

Suppression effects with $k_{\mathrm{CFP}}$ at 0.06 is better than that at 0.02

Suppression effects with $k_{\mathrm{CFP}}$ at 0.06 is worse than that at 0.02

(b)

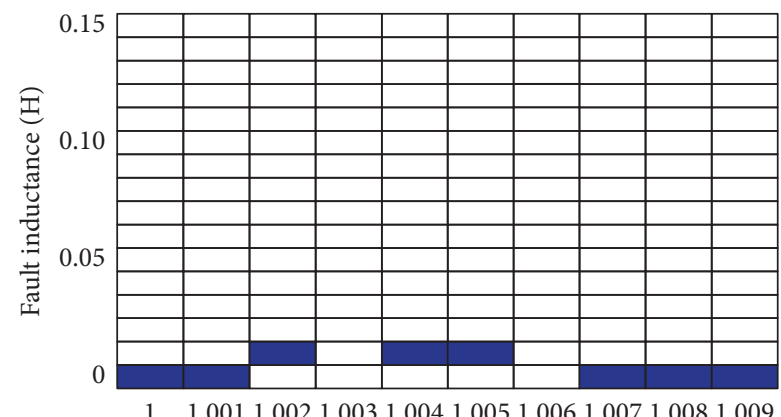

$1 \quad 1.0011 .0021 .0031 .0041 .0051 .0061 .0071 .0081 .009$ Fault starting time (s)

$\square$ Suppression effects with $k_{\mathrm{CFP}}$ at 0.06 and 0.02 are same

Suppression effects with $k_{\mathrm{CFP}}$ at 0.06 is better than that at 0.02

Suppression effects with $k_{\mathrm{CFP}}$ at 0.06 is worse than that at 0.02

(d)

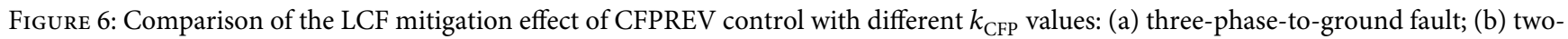
phase-to-ground fault; (c) line-to-line fault; (d) single-phase-to-ground fault. 


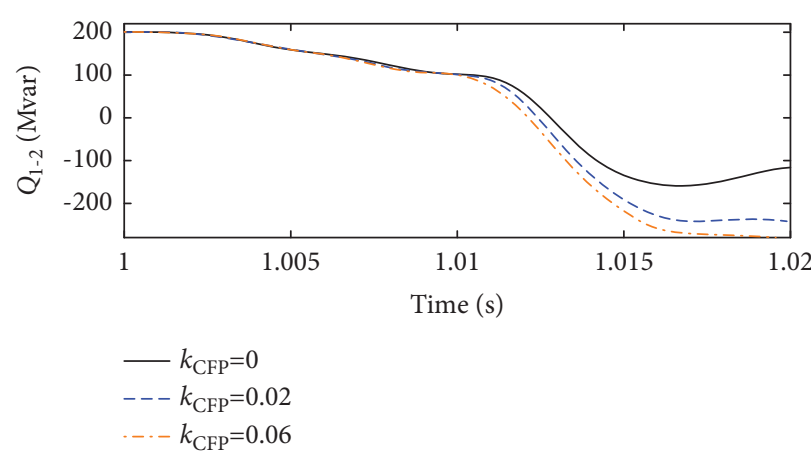

(a)

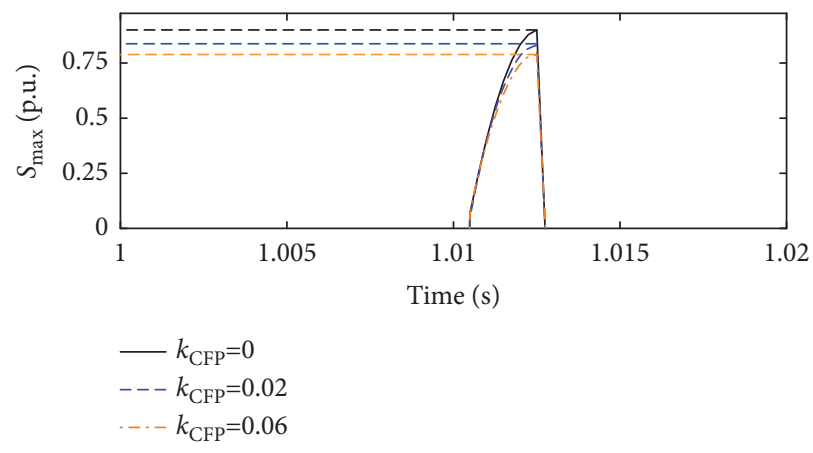

(c)

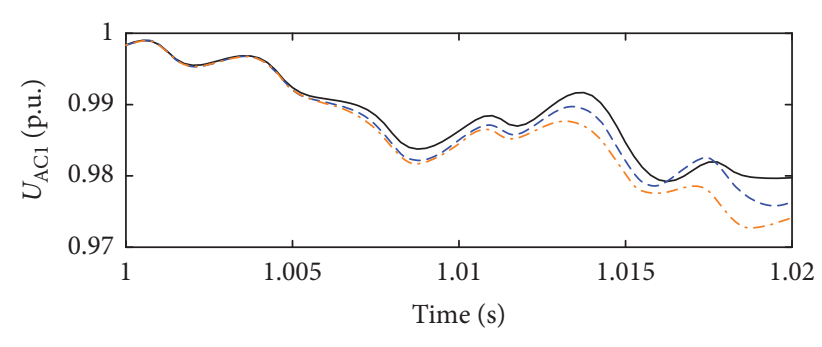

$$
\begin{aligned}
& -k_{\mathrm{CFP}}=0 \\
& ---k_{\mathrm{CFP}}=0.02 \\
& --k_{\mathrm{CFP}}=0.06
\end{aligned}
$$

(b)

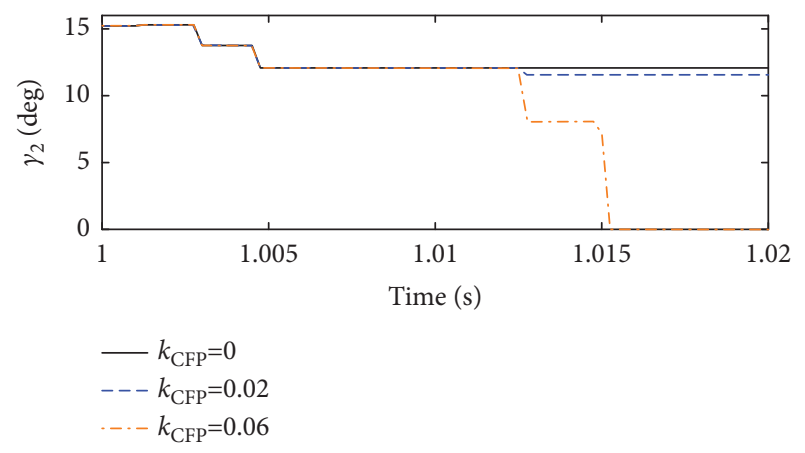

(d)

FIgURE 7: The impact of CFPREV control on commutation process in inverter 2. (a) Reactive power flows from inverter station 1 to 2; (b) RMS voltage of AC bus 2; (c) $S_{\max }$ of the inverter 2; (d) extinction angle of the inverter 2.

Figure 7. The fault starts at $1 \mathrm{~s}$ and lasts for $0.1 \mathrm{~s}$; the electrical distance from AC bus 1 is $0.1 \mathrm{H}$. It can be seen from Figure 7 (a) that in steady-state operation, AC system 1 delivers $Q_{1-2}$ of 200 Mvar to $A C$ system 2 via tie line. However, because of the voltage reduction of AC bus 1 after the occurrence of the fault, the reactive power is reversely transmitted from AC bus 2 to AC bus 1 for support. And the higher $k_{\mathrm{CFP}}$ in inverter 1 , the more reactive power is transmitted from AC bus 2 to 1 . As shown in Figures 7(b) and $7(\mathrm{c})$, transmitting more reactive power to AC system 1 will cause the insufficient reactive power of AC system 2 itself, and the descend of bus voltage will cause the shrink of $S_{\max }$, bringing the reduction of extinction angle to inverter 2 . When $\gamma$ decreases below $\gamma_{\text {min }}$, the CF occurs. The curve of $\gamma_{2}$ quickly falls to zero before $1.02 \mathrm{~s}$ in Figure $7(\mathrm{~d})$, indicating that the CFPREV control with $k_{\mathrm{CFP}}$ at 0.06 in inverter 1 leads to the CCF in inverter 2. And the impact of CFPREV control of inverter 1 on the commutation process of inverter 3 is illustrated in Figure 8, where the variation tendencies of the parameters are consistent with the previous analysis.

Table 3 gives a quantitative comparison of the parameters at $1.02 \mathrm{~s}$ under various fault scenarios to interpret the impact of $k_{\mathrm{CFP}}$ value of inverter 1 on the commutation process of remote inverter stations 2 and 3. The AC system faults with the inductance of $0.1 \mathrm{H}$ in Table 3 all occur at $1 \mathrm{~s}$ and last for $0.1 \mathrm{~s}$. The results of all types of fault in Table 3 are consistent with those in Figures 7 and 8. Comparing the $S_{\max }$ values as $k_{\mathrm{CFP}}$ rises from 0 to 0.06 , it can be observed that reactive power exchange $Q_{1-2}$ and $Q_{1-3}$ through tie line keeps going up, while $U_{\mathrm{AC}}$ and $S_{\max }$ keep decreasing, indicating that the larger $k_{\mathrm{CFP}}$ value leads to the high reactive power exchange from adjacent inverter stations and increases the possibility of CCF.

To sum up, when $k_{\mathrm{CFP}}$ is set as 0.06 , a larger output of CFPREV control of the inverter station near the fault is beneficial to the mitigation of LCF, whereas it is also likely to lead to the CCFs of remote stations due to the reactive power transfer between the stations through tie lines. Therefore, it is necessary to design a coordinated control scheme of CFPREV controls for multi-infeed UHVDC systems. This coordinated control scheme is hoped to not only effectively prevent its associated inverters from the LCF but also will not cause the CCF of adjacent UHVDC lines.

\section{Coordinated Control Scheme of CFPREVs in Multi-Infeed UHVDC System}

The coordinated control scheme of CFPREVs in a multiinfeed UHVDC system should satisfy the following two requirements:

(i) When the remote inverters have sufficient commutation margin (the difference value between realmeasured extinction angle and steady-state extinction angle), the CFPREV control of the inverter station near the fault should output a large advancement of firing angle for a better LCF mitigation effect 

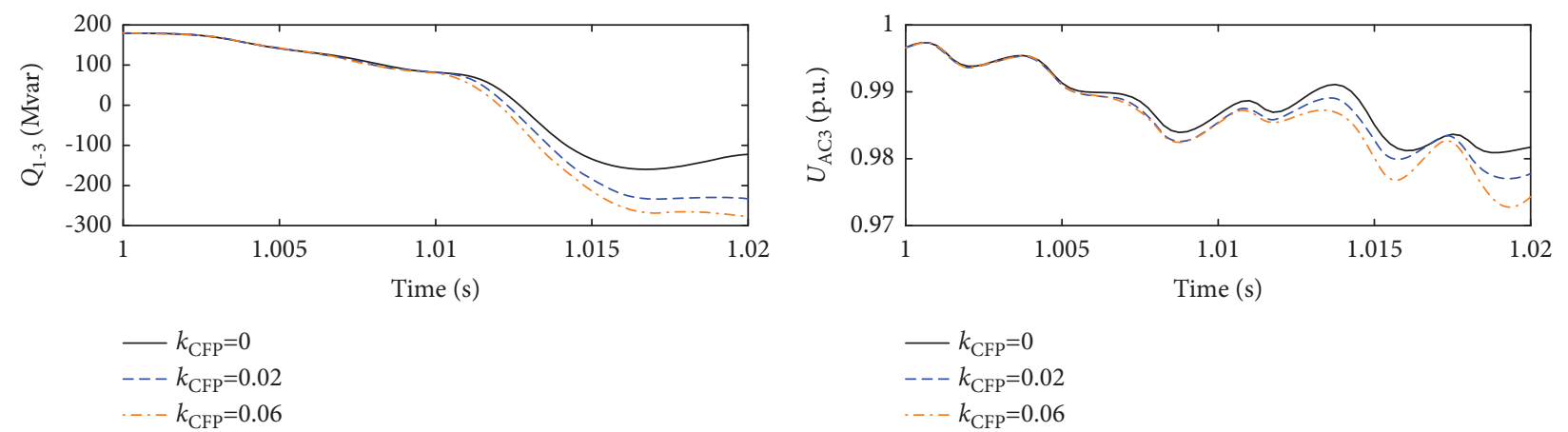

(a)

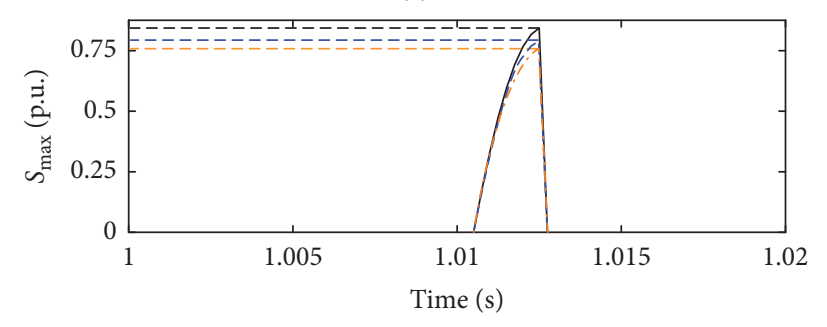

$$
\begin{aligned}
& -k_{\mathrm{CFP}}=0 \\
& ---k_{\mathrm{CFP}}=0.02 \\
& --k_{\mathrm{CFP}}=0.06
\end{aligned}
$$

(c)

(b)

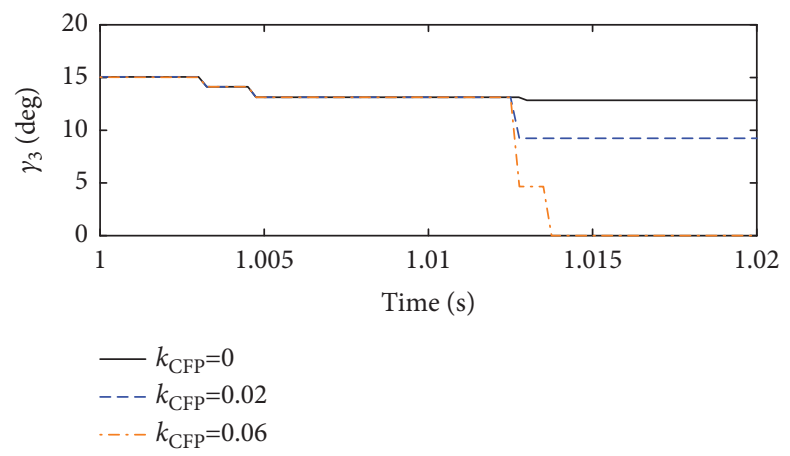

(d)

FIGURE 8: The impact of CFPREV control on commutation process in inverter 3. (a) Reactive power flows from inverter station 1 to 3; (b) RMS voltage of AC bus 3; (c) $S_{\max }$ of the inverter 3; (d) extinction angle of the inverter 3.

(ii) When the commutation margins of inverters in remote stations decrease obviously, the controller decreases the advancement of firing angle to diminish its influence on the commutation processes of remote stations

To meet the two requirements above, a coordinated control scheme of CFPREV controls shown in Figure 9 is proposed. The adaptive $k_{\mathrm{CFP}}\left(k_{\text {adap }}\right)$ control part can automatically adjust the value of $k_{\text {adap }}$ to change the output of CFPREV. Figure 9 shows the structure of coordinated control of CFPREV of inverter 1 as an example. The $k_{\text {adap }}$ shift control module contains two blocks, which are the coordinated control judgment block and the Switch block. And the coordinated control judgment block consists of two subunits, the voltage reduction detection subunit and the CFPREV initialization detection subunit. $S_{\mathrm{VOL}}$ is the enable signal of voltage reduction detection, and it will be set as 1 only when AC bus 1 is the near-fault bus whose voltage reduction is the most severe. In the CFPREV control enabling subunit, $\Delta \alpha_{\mathrm{CFP} 1}, \Delta \alpha_{\mathrm{CFP} 2}$, and $\Delta \alpha_{\mathrm{CFP} 3}$ are the output of CFPREV controls of inverters 1, 2, and 3, respectively. $S_{\mathrm{CFP}}$ is the enable signal of CFPREV initialization detection, and it will be set as 1 only when at least the CFPREV control of one station has been activated. The coordinated control judgment enable signal $S_{\mathrm{CO}}$ is 1 only when $S_{\mathrm{VOL}}$ and $S_{\mathrm{CFP}}$ are both 1. The Switch block mainly achieves changing $k_{\text {adap }}$ according to the maximum value of extinction angle reduction $\Delta \gamma_{\max }$ in remote inverter stations. The formula is shown as follows (the unit of the following formula is the electrical angle):

$$
k_{\text {adap }}= \begin{cases}k_{1}=k_{\text {sta }}, & \Delta \gamma_{\text {max }} \leq \frac{\Delta \gamma_{N},}{2}, \\ k_{2}=k_{\text {sta }}\left(1-\max \left\{\operatorname{HIIF}_{21}, \mathrm{HIIF}_{31}\right\}\right), & \frac{\Delta \gamma_{N}}{2}<\Delta \gamma_{\max } \leq \Delta \gamma_{N}, \\ k_{3}=k_{2}\left(1-\max \left\{\mathrm{HIIF}_{21}, \mathrm{HIIF}_{31}\right\}\right), & \Delta \gamma_{N}<\Delta \gamma_{\max } .\end{cases}
$$

According to engineering experience, the range of rated extinction angle $\gamma_{N}$ of the inverter is $\left[17^{\circ}, 22^{\circ}\right]$, and the steady-state control range of $\gamma_{N}$ in UHVDC transmission system is $\pm 2.5^{\circ}$. If the reduction of extinction angle $\Delta \gamma$ is more than $2.5^{\circ}$, there will be a risk that $\gamma$ continues decreasing rapidly and CF appears. As a result, the critical $\Delta \gamma$ of $k_{\text {adap }}$ shifting to the lowest value $k_{1}$ is set as $\gamma_{N}$, and the critical $\Delta \gamma$ of $k_{\text {adap }}$ shifting to the middle value $k_{2}$ is set as $\gamma_{N} / 2$. The values of $k_{1}$ and $k_{\text {sta }}$ are the constant value of $k_{\mathrm{CFP}}$ of the CFPREV originally installed in UHVDC lines. In the Henan multi-infeed system, the constant value of $k_{\mathrm{CFP}}$ in the CFPREV is set as 0.05 . The impact of the CFPREV on remote inverter stations is realized through the tie lies between inverter stations on the AC side. The strength of AC side coupling between station $i$ and $j$ is assessed by index $\operatorname{HIIF}_{j i}$ ranging from 0 to 1 . 
TABLE 3: Impact of $k_{\mathrm{CFP}}$ values on the inverter station far from the fault.

\begin{tabular}{|c|c|c|c|c|c|c|c|c|c|}
\hline \multirow{2}{*}{ Fault type } & \multirow{2}{*}{$k_{\mathrm{CFP}}$} & \multicolumn{4}{|c|}{ Parameters of inverter $2(t=1.02 \mathrm{~s})$} & \multicolumn{4}{|c|}{ Parameters of inverter $3(t=1.02 \mathrm{~s})$} \\
\hline & & $Q_{1-2}$ (Mvar) & $U_{\mathrm{AC} 2}$ (p.u.) & $S_{\max 2}$ (p.u.) & $\gamma_{2}(\operatorname{deg})$ & $Q_{1-3}$ (Mvar) & $U_{\mathrm{AC} 3}$ (p.u.) & $S_{\max 3}$ (p.u.) & $\gamma_{3}(\mathrm{deg})$ \\
\hline \multirow{3}{*}{ LLLG fault } & 0 & -103 & 0.980 & 0.92 & 12.5 & -110 & 0.982 & 0.88 & 13.5 \\
\hline & 0.02 & -254 & 0.976 & 0.86 & 11.8 & -216 & 0.978 & 0.83 & 9.1 \\
\hline & 0.06 & -315 & 0.973 & 0.79 & $\mathrm{CCF}$ & -291 & 0.974 & 0.77 & $\mathrm{CCF}$ \\
\hline \multirow{3}{*}{ LLG fault } & 0 & -125 & 0.975 & 0.89 & 10.4 & -135 & 0.979 & 0.91 & 11.7 \\
\hline & 0.02 & -250 & 0.972 & 0.84 & 9.4 & -240 & 0.976 & 0.89 & 11.0 \\
\hline & 0.06 & -280 & 0.970 & 0.82 & 9.1 & -275 & 0.974 & 0.83 & 9.3 \\
\hline \multirow{3}{*}{ LL fault } & 0 & -31 & 0.982 & 0.91 & 10.3 & -50 & 0.983 & 0.93 & 11.5 \\
\hline & 0.02 & -100 & 0.979 & 0.89 & 8.5 & -110 & 0.980 & 0.89 & 10.2 \\
\hline & 0.06 & -150 & 0.975 & 0.84 & 6.0 & -168 & 0.978 & 0.86 & 8.7 \\
\hline \multirow{3}{*}{ SLG fault } & 0 & 0 & 0.988 & 0.93 & 11.3 & -5 & 0.989 & 0.95 & 11.8 \\
\hline & 0.02 & -80 & 0.986 & 0.80 & 11.0 & -70 & 0.987 & 0.91 & 10.5 \\
\hline & 0.06 & -100 & 0.983 & 0.88 & 10.5 & -110 & 0.985 & 0.87 & 8.2 \\
\hline
\end{tabular}

$$
\mathrm{HIIF}_{j i}=\left|\frac{Z_{\text {eqji }}}{Z_{\text {eqii }}}\right|,
$$

where $Z_{\text {eqii }}$ and $Z_{\text {eqji }}$ are corresponding elements in the equivalent impedance matrix. Consequently, it can be defined that the AC side coupling distance between the inverter station $j$ far from the fault and the inverter station $i$ near the fault is

$$
d_{j i}=\left|1-\operatorname{HIIF}_{j i}\right| .
$$

Apparently, the stronger the interaction between to inverter station is, the greater $\operatorname{HIIF}_{j i}$ is, the smaller distance $d_{j i}$ is, the smaller value of the coefficient $k_{\text {adap }}$ should be. The values of $k_{2}$ and $k_{3}$ are determined by the closest interaction distance $d_{j i}$. According to the parameter determination method interpreted above, the parameters applied in this manuscript are as follows:

$$
k_{\text {adap }}= \begin{cases}0.05, & \Delta \gamma_{\max } \leq 1.25^{\circ}, \\ 0.035, & 1.25^{\circ}<\Delta \gamma_{\max } \leq 2.5^{\circ}, \\ 0.0245, & 2.5^{\circ}<\Delta \gamma_{\max } .\end{cases}
$$

Note that because this control scheme is applied in the AC fault transient process, the determination method of the control parameters above can only be a reference. For a given multi-infeed system, verification and adjustment of the parameters in (19) based on the simulation are required.

Figure 10 is the flow chart of the coordinated control scheme of CFPREVs in a multi-infeed UHVDC system. The reference values $\gamma_{\text {stai }}, \Delta \gamma_{\text {th }}$, and $U_{\text {stai }}$ need to be input in advance, and they will be compared with the real-time measured parameters in the transient process to evaluate the operation condition of inverter stations. As the coefficient of coordinated CFPREV control under normal operation, $k_{\text {sta }}$ also needs to be set in advance. Firstly, the coordinated controller keeps monitoring the extinction angle drop of the inverters in remote stations. When $\Delta \gamma_{\max }$ is larger than the threshold $\Delta \gamma_{\text {th }}$ in (17), the controller will check the value of $S_{\mathrm{CO}}$. If $S_{\mathrm{CO}}$ is 1 , that means the fault occurs near this inverter station, and its corresponding activated CFPREV controller is threatening the commutation process in remote stations. Then, the coordinated control scheme of CFPREVs will switch to $k_{\text {adap }}$ to adjust the output of CFPREV.

\section{Simulation Verification}

The correct actions of the coordinated controller of CFPREVs should include the following:

(i) The coordinated controller of CFPREVs can identify the nearest UHVDC line to the fault

(ii) The coordinated controller of CFPREVs installed in the inverter station near the fault can adaptively switch the CFPREVk according to the extinction angle reduction values of remote stations based on the design requirements

4.1. Effectiveness of Coordinated CFPREV Controls Strategy. The effectiveness of the coordinated control strategy of CFPREV is also verified in Henan provincial multi-infeed UHVDC system. According to the actual construction parameters, the fiber distance between $\mathrm{AC}$ buses 2 and 3 is negligible, and the fiber path distance between AC buses 1 and 2 is about $200 \mathrm{~km}$. In terms of current technology, considering the delay of additional equipment such as power amplifiers, the transmission rate of optical fiber communication is $120-200 \mathrm{~km} / \mathrm{ms}$ [32]. So, the transmission delay of the coordinated CFPREV for inverter 1 is considered as $1.5 \mathrm{~ms}$. In this case, the commutation performances in the tested system with coordinated CFPREV and traditional CFPREV with a fixed coefficient $k_{\mathrm{CFP}}$ at 0.05 are compared.

The performance of the coordinated controller of CFPREV on mitigating CCF under a three-phase-to-ground symmetric fault case is indicated in Figure 11, where the fault with $0.18 \mathrm{H}$ inductance is set near the AC bus 1 , occurring at $1 \mathrm{~s}$ and lasting for $0.1 \mathrm{~s}$. As shown in Figure 11(b), with coordinated control of CFPREV, the coefficient $k_{\text {adap }}$ decreases from 0.05 to 0.035 and then to 0.0245 when detecting the reduction of extinction angle in adjacent station $\Delta \gamma_{\max }$ keeps rising. And the firing angle advancement order $\Delta \alpha_{\text {CFP }}$ in Figure $11(\mathrm{~g})$ will also decrease. According to equation (17), $Q_{d}$ increases as the reduction of $\alpha$. Therefore, with a smaller output of coordinated CFPREV control $\Delta \alpha_{\mathrm{CFP}}$, the reactive consumption of the inverter station near the fault will be smaller due to a larger $\alpha$. As the decrease of reactive consumption of inverter near the fault, the voltage dip of its 


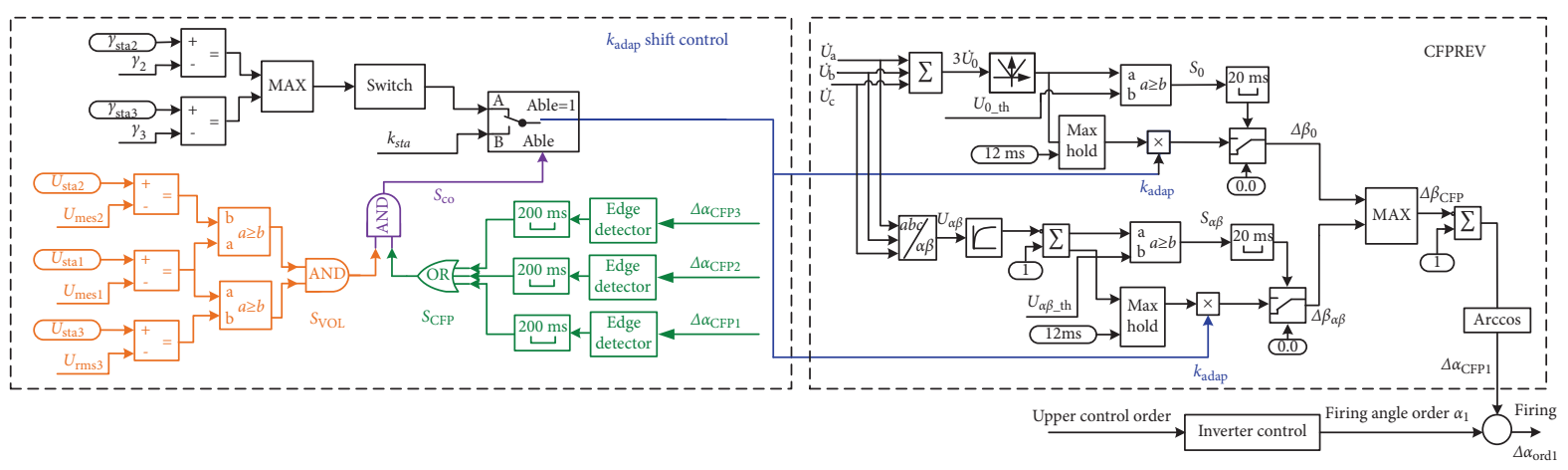

FIGURE 9: Structure of coordinated control scheme of CFPREV.

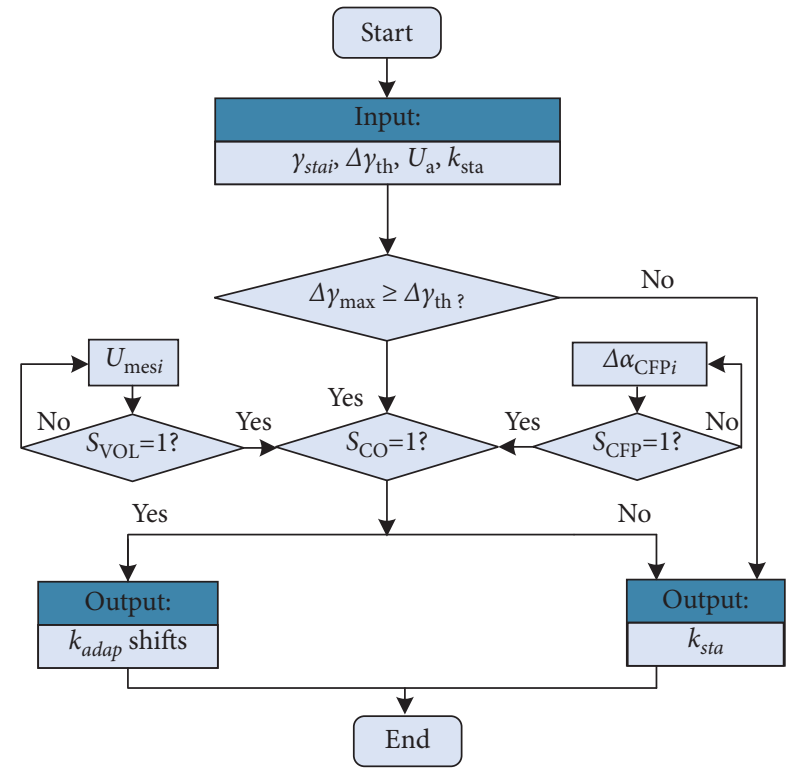

Figure 10: Flow chart of the coordinated control scheme of CFPREVs.

associated AC bus will also be lighter. Consistently, the reactive support from the adjacent inverter station will also decrease, and the voltage dip of the adjacent AC bus is caused by the reactive power exchange though the AC side is lighter. The values of $S_{\max 2}$ and $S_{\max 3}$ in Figures 11(c) and 11 (d) with coordinated control are obviously larger than those with traditional control, showing that inverters 2 and 3 have more sufficient commutation margin under coordinated control. According to equation (19), the extinction angle will increase as $\Delta U_{i}$ decreases, and this analysis is verified in Figures 11(e) and 11(f). The black curves of $\gamma_{2}$ and $\gamma_{3}$ all fall to zero, indicating that the CCF occurs in both inverters 2 and 3 with traditional CFPREV control, while the lowest values of blue curves all stay above $10^{\circ}$, showing that inverters 2 and 3 are prevented from the CCF under coordinated control. So the coordinated controller can adaptively adjust $k_{\text {adap }}$ to change the CFPREV output according to $\Delta \gamma_{\max }$. And it can be seen from the curve in Figure 11(h) that because inverters 2 and 3 of UHVDC system 2 are prevented from CCFs by coordinated CFPREV, the reduction of the DC power transmission $P_{2}$ is significantly lighter. The performance of the coordinated controller of CFPREV on mitigating CCF under two-phase-to-ground asymmetric fault case is indicated in Figure 12, where the fault with $0.06 \mathrm{H}$ inductance is also set near AC bus 1, occurring at $1 \mathrm{~s}$ and lasting for $0.1 \mathrm{~s}$. The results in Figure 12 are consistent with those in Figure 11, and the coordinated controller of CFPREV also successfully mitigates the CCFs in remote inverter stations 2 and 3. Most of the CFs in the HVDC transmission system occur in the inverter station, caused by the short-circuit fault in the AC system on receiving side. Therefore, the effectiveness of the CF mitigation method also should be verified under various short-circuit fault cases. Since the single-phase-to-ground fault and lineto-line fault will not lead to CCF in remote inverter stations in the simulation of the Henan multi-infeed system, only results under three-phase-to-ground symmetric fault and two-phase-to-ground asymmetric fault cases are indicated here.

Figure 13 compares the effectiveness of coordinated CFPREV and traditional CFPREV with CFPREVk of 0.05. As the blue boxes indicated in the figure, the coordinated CFPREV greatly reduces the probability of CCFs in inverters 2 and 3 caused by CFPREV and consequently decreases the impact of CFPREV on the commutation process of the inverters in adjacent lines in the UHVDC multi-infeed system.

Based on the analysis above, when a $1.5 \mathrm{~ms}$ transmission delay time is considered, the coordinated controller can timely reduce the output of CFPREV of UHVDC system 1 after detecting $\gamma_{2}$ and $\gamma_{3}$ to reach the threshold value, thus diminishing its influence on the commutation process of inverters 2 and 3 in UHVDC system 2.

\subsection{Impact of Transmission Delay on Coordinated CFPREV} Strategy. According to the simulation results, if the CCFs of adjacent UHVDC links occur, the interval between the occurrence of CCFs and the initiation of AC fault is never beyond $20 \mathrm{~ms}$. Therefore, the impact of transmission delay on the performance of the coordinated CFPREV should be considered in engineering practice. Through the simulation, it is found that the acceptable transmission delay limit of the coordinated CFPREV control strategy proposed in this paper is $4.5 \mathrm{~ms}$. In other words, when the distance between two converter stations in a multi-infeed system is within $600 \mathrm{~km}$ (delay time less than $4.5 \mathrm{~ms}$ ), this control strategy is 


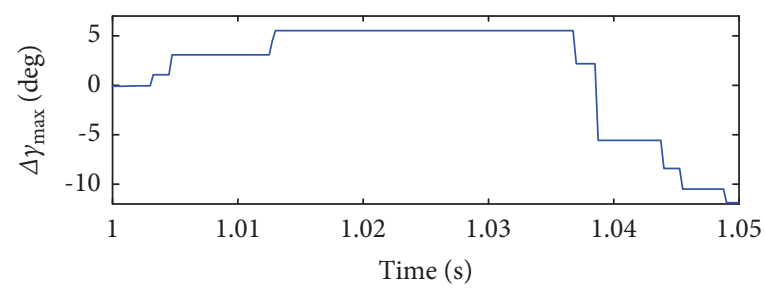

(a)

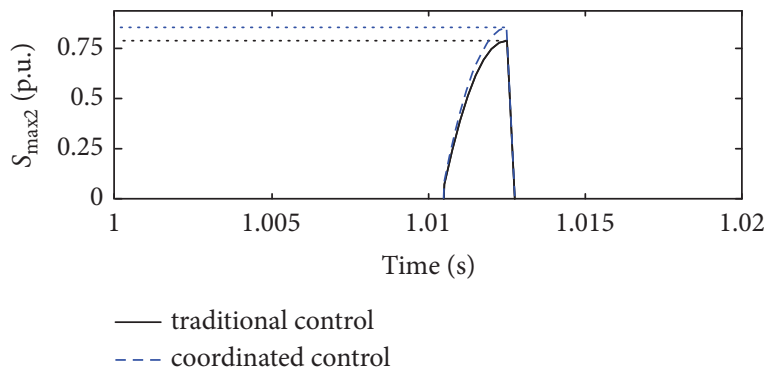

(c)

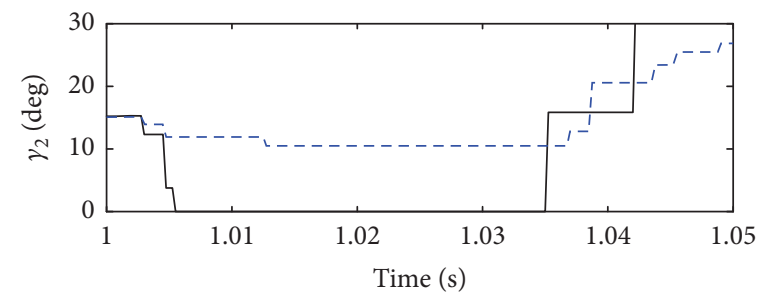

— traditional control

- - - coordinated control

(e)

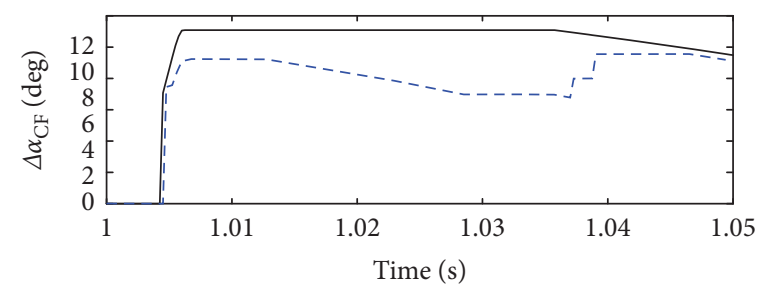

- traditional control

- - - coordinated control

(g)

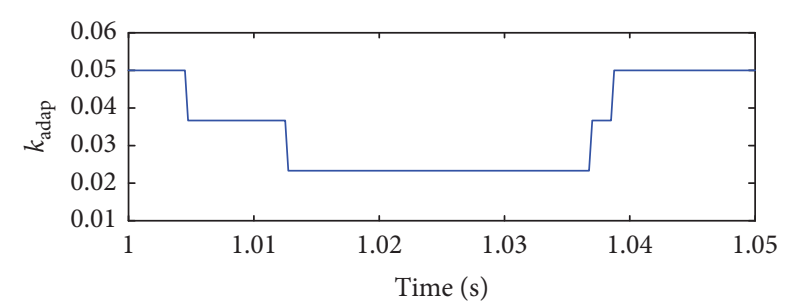

(b)

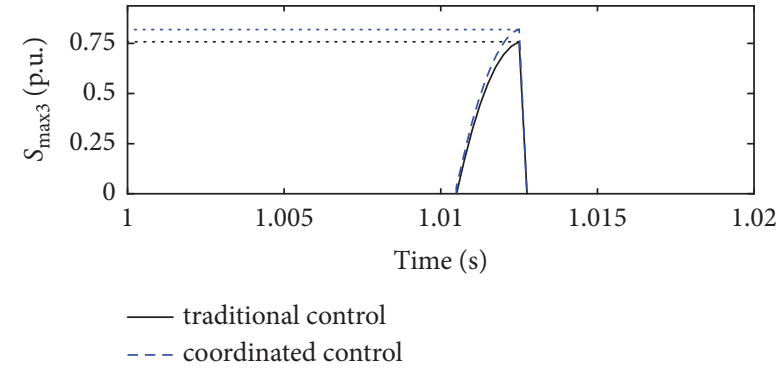

(d)

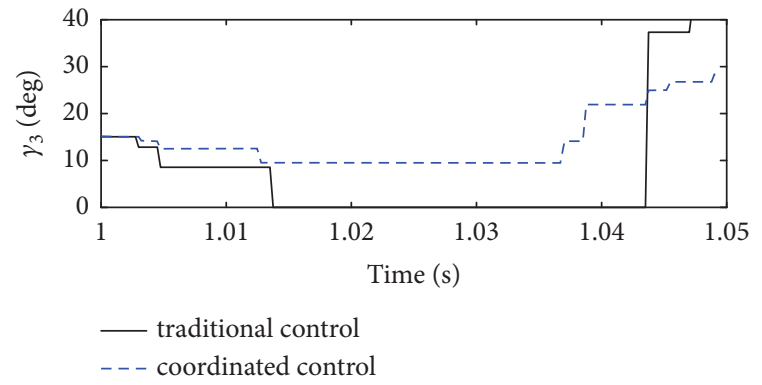

(f)

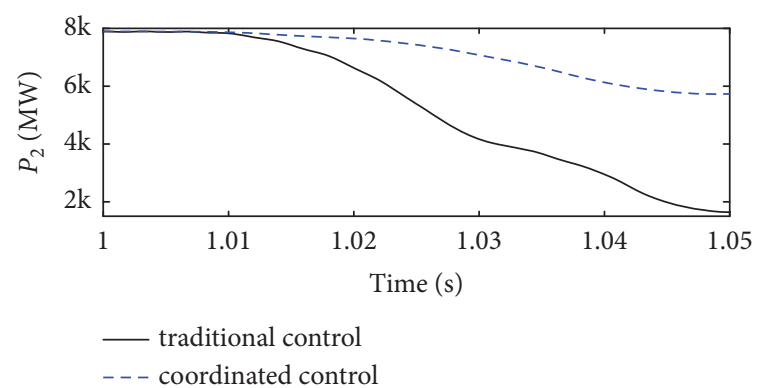

(h)

FIgURE 11: Performance of the coordinated controller of CFPREV under three-phase-to-ground symmetric fault case. (a) Maximum reduction value of the extinction angles among inverters 2 and 3 ; (b) adjustment process of the coefficient $k_{\text {adap }}$; (c) comparison of $S_{\max 2}$; (d) comparison of $S_{\max 3}$; (e) comparison of $\gamma_{2}$; (f) comparison of $\gamma_{3}$; (g) comparison of CFPREV output; (h) comparison of $P_{2}$.

applicable. The distances between the inverter stations in actual multi-infeed systems are quite close, and the distance limit above is generally satisfied. For example, the fast fiber path distance between the converter buses in Henan provincial multi-infeed UHVDC system is about $200 \mathrm{~km}$. Figure 14 gives a comparison about the performances of coordinated CFPREV strategy under different transmission time delays when a three-phase-to-ground fault with the inductance of $0.20 \mathrm{H}$ is set near inverter 1 .

According to Figure 14(a), when the time delay is considered as $4.5 \mathrm{~ms}$ and $5 \mathrm{~ms}$, the moment when $k_{\text {adap }}$ switches from 0.05 to a smaller value is retarded. And in
Figure 14(b), the change of $\Delta \gamma_{\max }$ received by coordinated CFPREV in inverter station 1 is also deferred. Figures 14(c) and $14(\mathrm{~d})$ indicate that inverters 2 and 3 will not suffer CCFs until the transmission delay increases to $5 \mathrm{~ms}$.

4.3. Impact of Noise Interference on Coordinated CFPREV Strategy. During the operation process of the coordinated CFPREV, the sampling, transformation, processing, sending, transmission, and receiving of electrical signals will be confronted with noise interference. And noise interference will change the performance of the coordinated CFPREV 


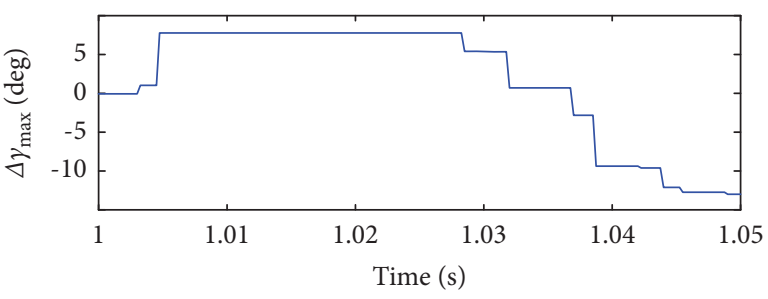

(a)

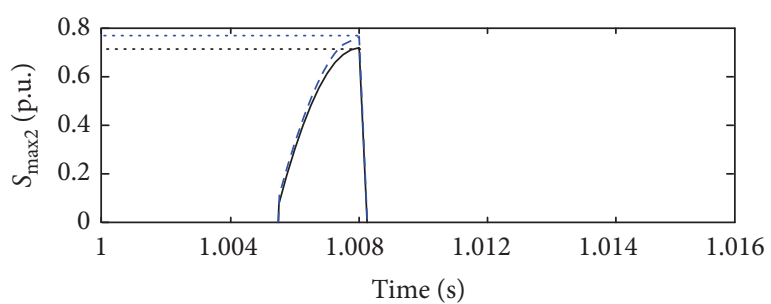

— traditional control

- - - coordinated control

(c)

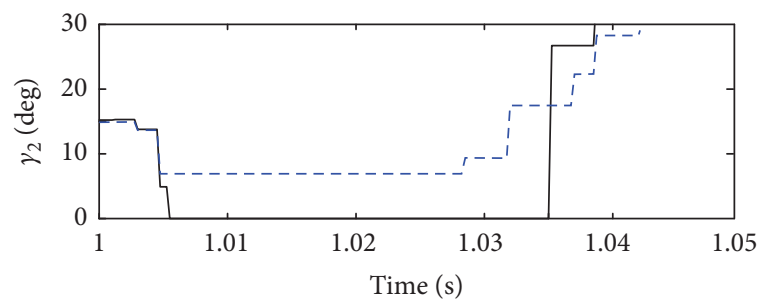

— traditional control

- - - coordinated control

(e)

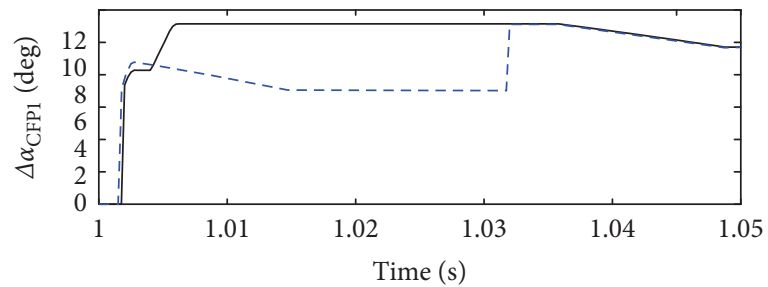

— traditional control

- - - coordinated control

(g)

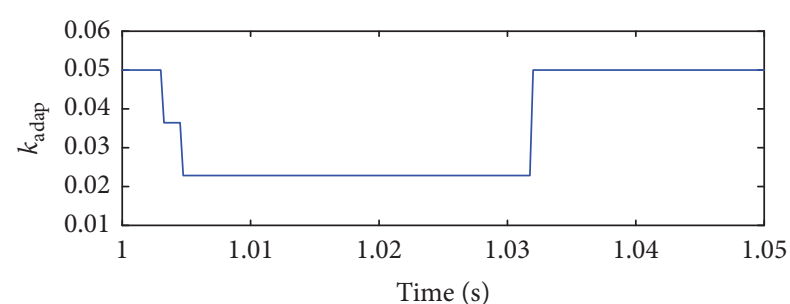

(b)

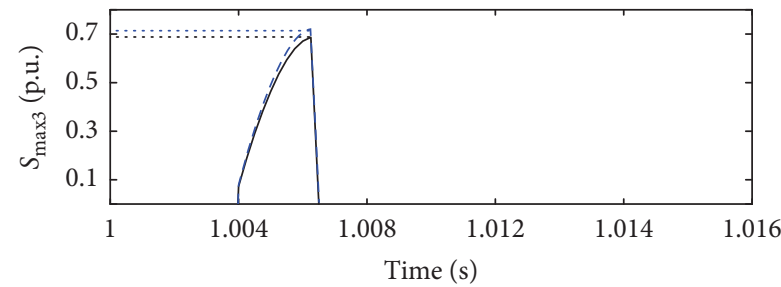

_ traditional control

- - - coordinated control

(d)

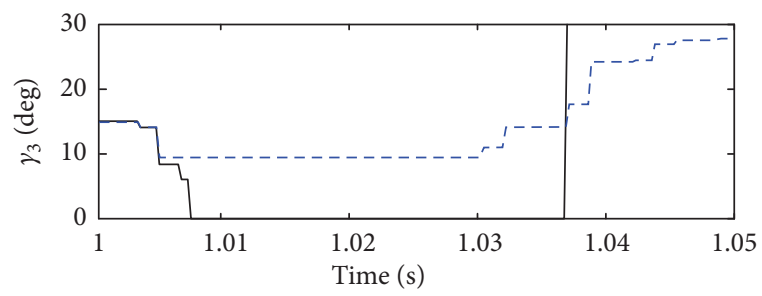

_ traditional control

- - - coordinated control

(f)

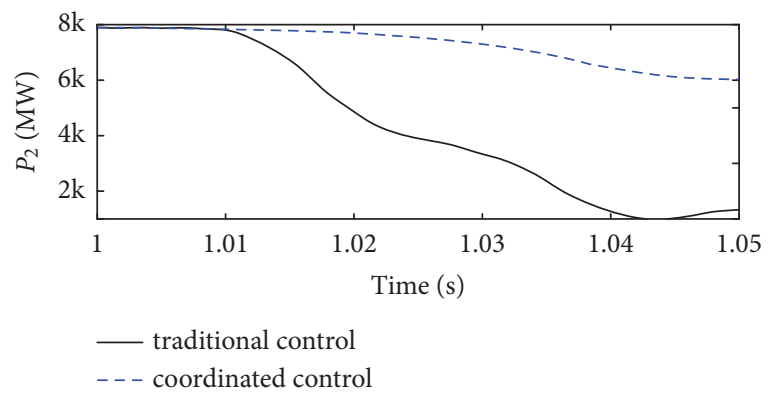

(h)

FIgURE 12: Performance of the coordinated controller of CFPREV under two-phase-to-ground asymmetric fault case. (a) Maximum reduction value of the extinction angles among inverters 2 and 3; (b) adjustment process of the coefficient $k_{\text {adap }}$; (c) comparison of $S_{\max 2}$; (d) comparison of $S_{\max 3}$; (e) comparison of $\gamma_{2}$; (f) comparison of $\gamma_{3}$; (g) comparison of CFPREV output; (h) comparison of $P_{2}$.

controller and consequently affect the effectiveness of CCF mitigation. In order to test the sensitivity of the proposed strategy to noise and give a brief reference to the engineering application, a random signal noise within a certain amplitude is added to the instantaneous three-phase AC voltage sampling block of CFPREV (the right half part of Figure 9). And the test results are shown in Figure 15, where a threephase-ground fault with an inductance of $0.1 \mathrm{H}$ is set at $1 \mathrm{~s}$ and lasts for $0.1 \mathrm{~s}$ near inverter station 1 .

As shown in Figure 15(a), the noisesensitive tests include four groups, which are non-noise interference group (noise ratio $0 \%$, and the noise signal ratio of $1 \%, 2 \%$, and $3 \%$ groups. For example, in the test group of noise ratio $1 \%$, the maximum voltage signal sent to the control circuit on the secondary side is $5 \mathrm{~V}$ after transformation. Then, the random signal generator will generate a random number within the range of $[-0.05,0.05]$ every $50 \mathrm{~ms}$ to add the voltage signal. Note that, in this test, noise signals are added to each phase of the AC voltage. According to Figures 15(b)-15(d), the curves of control order in $0 \%$ and $1 \%$ noise ratio groups are basically coincident, indicating that the control process of coordinated CFPREV can still operate correctly to mitigate 


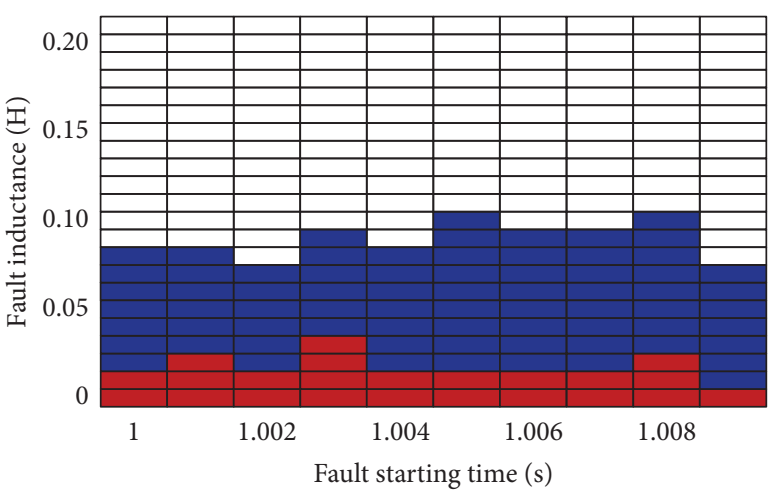

CCFs in inverters 2 or 3 are mitigated under CFPREV coordinated control

CCFs occur in inverters 2 or 3 under coordinated CFPREV control

(a)

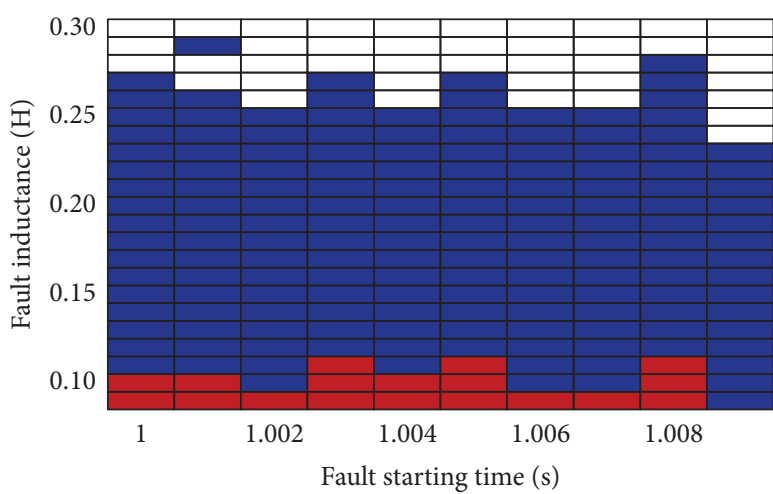

CCFs in inverters 2 or 3 are mitigated under CFPREV coordinated control

CCFs occur in inverters 2 or 3 under coordinated CFPREV control

(b)

FIGURE 13: Comparison of the effectiveness of coordinated and traditional CFPREV controls. (a) Two-phase-to-ground asymmetric fault; (b) three-phase-to-ground symmetric fault.

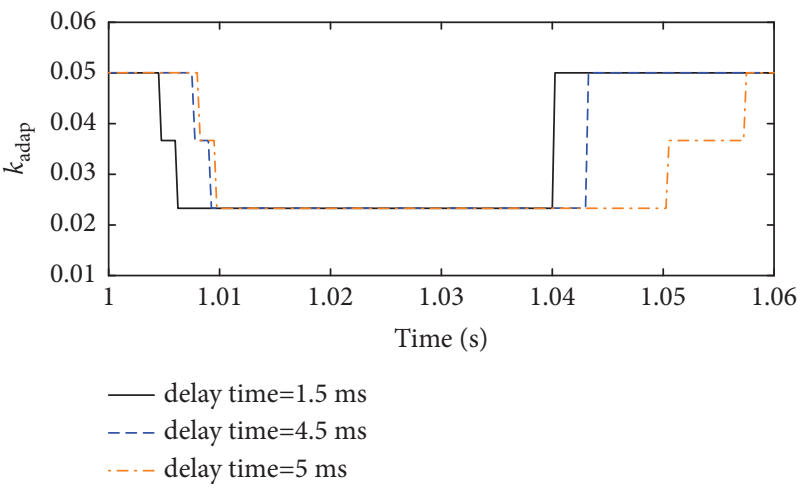

(a)

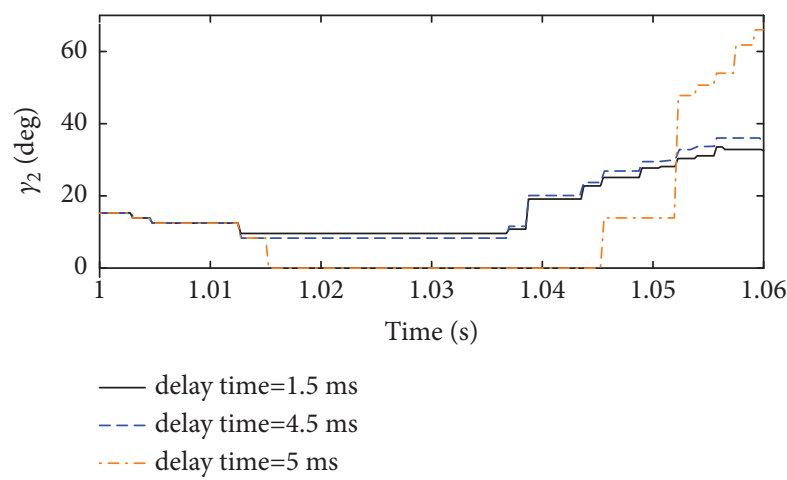

(c)

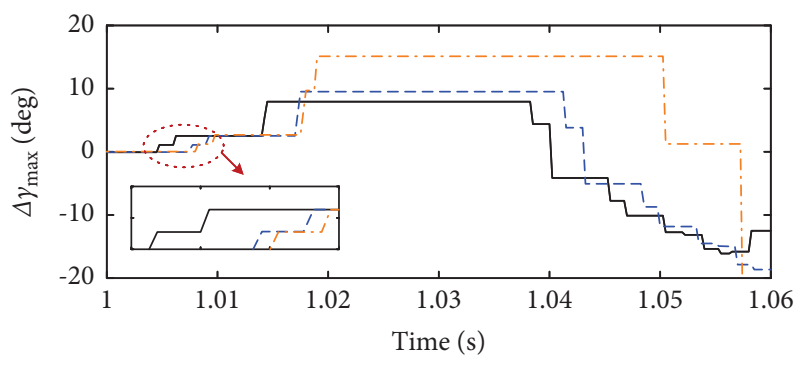

- delay time $=1.5 \mathrm{~ms}$

-- delay time $=4.5 \mathrm{~ms}$

-..- delay time $=5 \mathrm{~ms}$

(b)

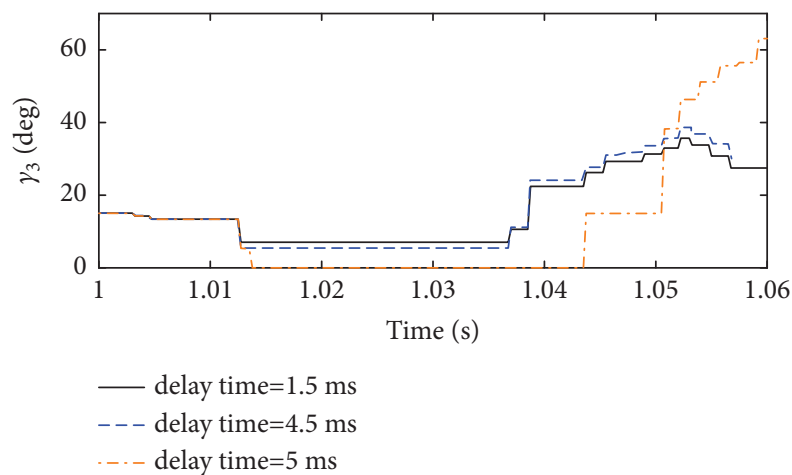

(d)

FIGURE 14: Comparison of the effectiveness of coordinated CFPREV control strategy with the delay time of $1.5 \mathrm{~ms}, 4.5 \mathrm{~ms}$, and $5 \mathrm{~ms}$. (a) Comparison of the switch of $k_{\text {adap }}$; (b) comparison of the detected $\Delta \gamma_{\max }$; (c) comparison of $\gamma_{2}$; (d) comparison of $\gamma_{3}$.

CCF within the range of $1 \%$ noise ratio. However, in the $2 \%$ and $3 \%$ noise ratio groups, the curves of control order are shifted. The coordinated CFPREV control can no longer give the firing order timely and accurately under these circumstances so that CCF occurs in the remote inverters 2 and 3.
Because the effectiveness of CF prevention relies on timeliness and accuracy, the coordinated control scheme of CFPREVs proposed in this paper is quite sensitive to noise. And the noise ratio above $1 \%$ added to the voltage signal will reduce the effectiveness of the controller. Noise sources in 


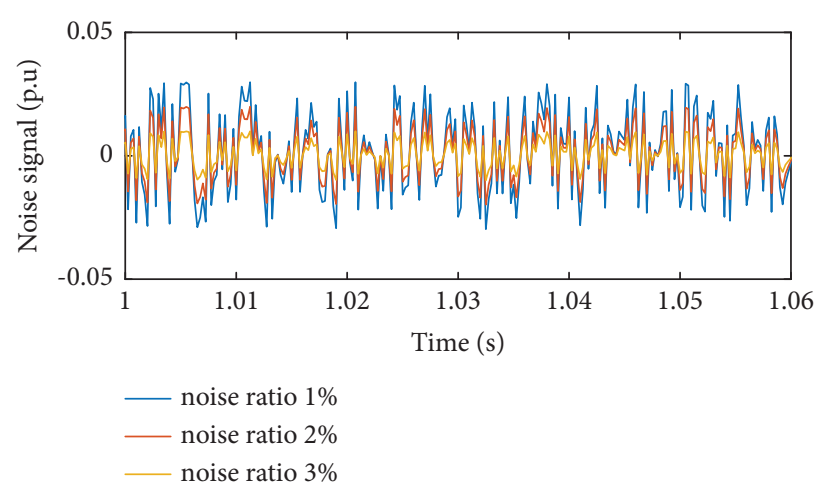

(a)
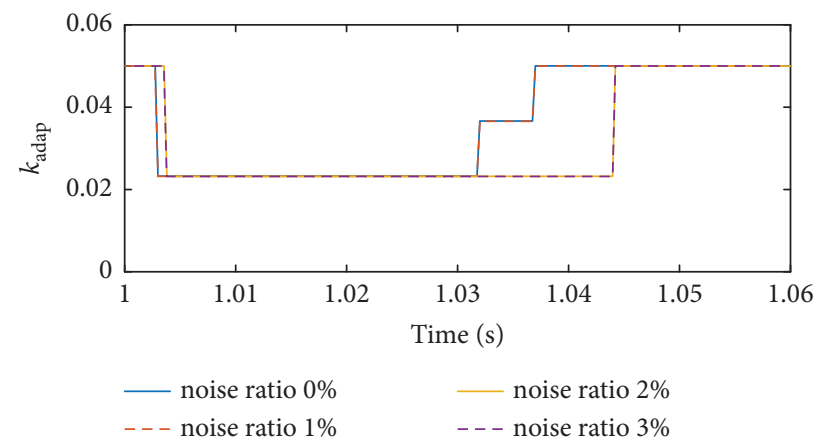

(c)

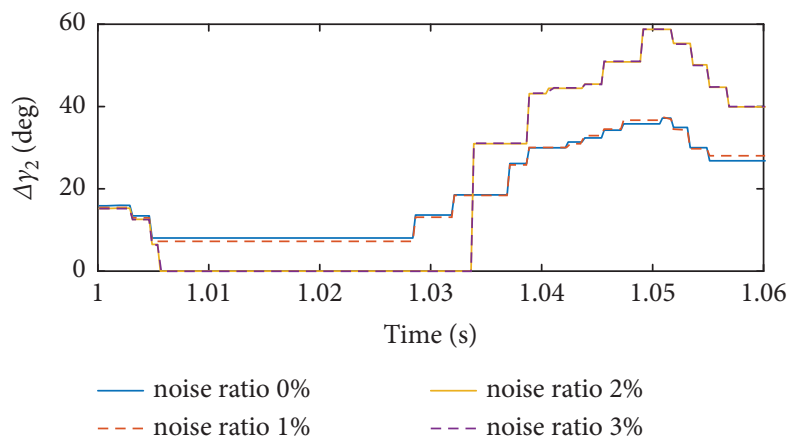

(e)
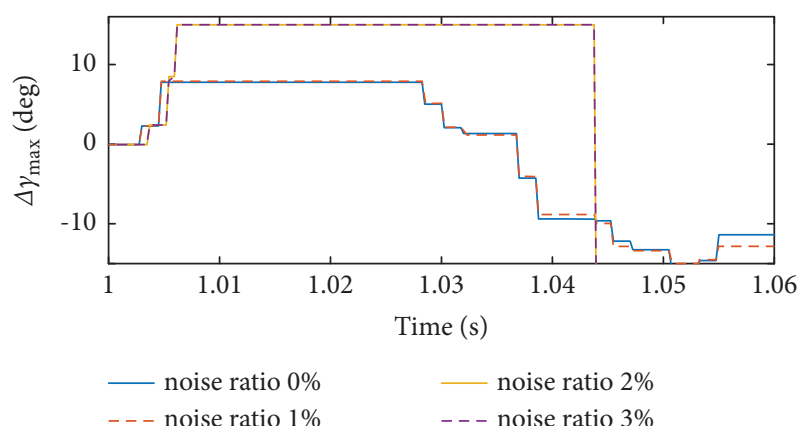

(b)

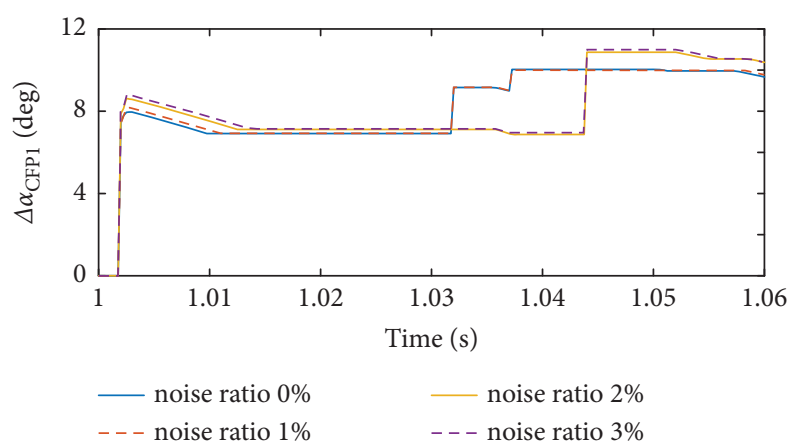

(d)

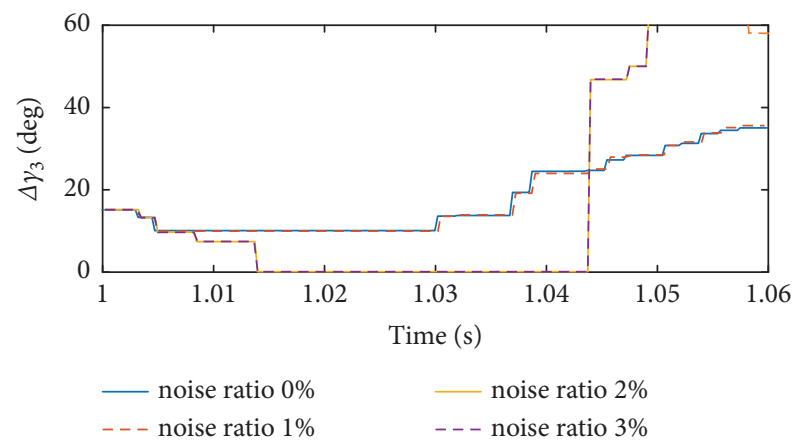

(f)

FIGURE 15: Comparison of the effectiveness of coordinated and traditional CFPREV controls. (a) Random noise signal; (b) maximum reduction value of the extinction angles; (c) adjustment process of the coefficient $k_{\text {adap }}$; (d) CFPREV output; (e) extinction angle of inverter 2; (f) extinction angle of inverter 3.

electrical power systems are complex, including the operational noise caused by the switching of electrical equipment, the noise of electromagnetic and common impedance coupling, the noise caused by transformer substation operation, and the noise caused by the large-scale integrated circuit. Therefore, antinoise interference is the problem that must be solved in the practical application of the coordinated control scheme of CFPREVs controller, and it is also the main task in the following research for authors.

\section{Conclusions}

In this paper, the impact of CFPREV control on the commutation process of UHVDC lines in the multi-infeed system is expounded. On the one hand, CFPREV control indeed effectively mitigates LCF by advancing the firing instantly. On the other hand, due to the reactive power exchanged between the stations, CFPREV control also further decreases the voltages of adjacent AC buses and consequently leads to the CCFs in the multi-infeed system. To mitigate this adverse impact, a coordinated control scheme of CFPREVs is proposed for a multi-infeed UHVDC system. The coordinated controller can adaptively adjust the coefficient $k_{\text {adap }}$ to change the output of CFPREV according to the extinction angle reduction of the inverters in remote stations. When the remote inverters have sufficient commutation margin, the CFPREV control of the inverter station near the fault will output a large advancement order of firing angle for a better LCF mitigation effect. When the commutation margins of inverters in remote stations decrease to a critical level, the controller decreases the advancement of firing angle to diminish the probability of 
CCF. Because the effectiveness of CF prevention control relies on timely and accurate signal transmission and analysis, the sensitivity of this method to transmission delay and noise is also discussed. The acceptable transmission delay of this coordinated strategy is within $4.5 \mathrm{~ms}$, which can usually be satisfied in the actual project because the distance between converter stations in a multi-infeed system is quite close (within $600 \mathrm{~km}$ ). The coordinated control scheme of CFPREVs is quite sensitive to noise. And the effectiveness of the controller will be affected when the noise ratio added to the analog signal reaches $2 \%$. Finally, the effectiveness of the coordinated CFPREV control strategy considering transmission delay and noise is verified under various fault cases by simulation.

However, the effectiveness of the proposed coordinated CFPREV strategy depends on the correct and timely transmission of remote converter operation signals. Its essence is a kind of wide area measurement protection. If the signals are lost or interfered with or error occurs during transmission, the coordinated CFPREV controller may refuse or incorrectly operate, so that the state of the system deteriorates. Consequently, the authors will further consider the treatment under the signal error or loss situation in future work.

\section{Abbreviations}

CCF: Concurrent commutation failure

CFPREV: Commutation failure prevention

LCC- Line-commutated converter high-voltage DC

HVDC:

LCF: $\quad$ Local commutation failure

UHVDC: Ultrahigh-voltage DC.

\section{Data Availability}

Access to data is restricted.

\section{Conflicts of Interest}

The authors declare that they have no conflicts of interest.

\section{Acknowledgments}

This work was supported by the Science and Technology Project of Hubei Provinces, State Grid Corporation of China (SGHB0000DKJS2104209).

\section{References}

[1] H. Zhou, W. Yao, X. Ai, D. Li, J. Wen, and C. Li, "Comprehensive review of commutation failure in HVDC transmission systems," Electric Power Systems Research, vol. 205, Article ID 107768, 2022.

[2] J. Zhao, R. Zeng, Q. Jiang, and B. Li, "Coordinated DC voltage control strategy of hybrid HVDC with multi-infeed MMC inverters considering commutation failures," International Transactions on Electrical Energy Systems, vol. 31, Article ID e12881, 2021.

[3] L. Hong, X. Zhou, H. Xia, Y. Liu, and A. Luo, "Mechanism and prevention of commutation failure in LCC-HVDC caused by sending end AC faults," IEEE Transactions on Power Delivery, vol. 36, no. 1, pp. 473-476, 2021.

[4] W. Wang, X. Xiong, M. Li, and R. Yu, "A flexible control strategy to prevent sending-end power system from transient instability under HVDC repetitive commutation failures," IEEE Transactions on Power Systems, vol. 35, no. 6, pp. 4445-4458, 2020.

[5] D. Huang, Y. Shu, J. Ruan, and Y. Hu, "Ultra high voltage transmission in China: developments, current status and future prospects," Proceedings of the IEEE, vol. 97, no. 3, pp. 555-583, 2009.

[6] L. Zhang and L. Dofnas, "A novel method to mitigate commutation failures in HVDC systems," in Proceedings of the International Conference on Power System Technology, pp. 51-56, Kunming, China, October 2002.

[7] X. Peng, W. Yao, C. Yan, J. Wen, and S. Cheng, "Two-stage variable proportion coefficient based frequency support of grid-connected DFIG-WTs," IEEE Transactions on Power Systems, vol. 35, no. 2, pp. 962-974, 2020.

[8] Q. Wang, W. Yao, J. Fang et al., "Dynamic modeling and small signal stability analysis of distributed photovoltaic gridconnected system with large scale of panel level DC optimizers," Applied Energy, vol. 259, Article ID 114132, 2020.

[9] Z. Wei, Y. Yuan, X. Lei, H. Wang, G. Sun, and Y. Sun, "Directcurrent predictive control strategy for inhibiting commutation failure in HVDC converter," IEEE Transactions on Power Systems, vol. 29, no. 5, pp. 2409-2417, 2014.

[10] H. Xiao, Y. Li, and X. Duan, "Enhanced commutation failure predictive detection method and control strategy in multi-infeed LCC-HVDC systems considering voltage harmonics," IEEE Transactions on Power Systems, vol. 36, no. 1, pp. 81-96, 2021.

[11] E. Rahimi, A. M. Gole, J. B. Davies, I. T. Fernando, and K. L. Kent, "Commutation failure analysis in multi-infeed HVDC systems," IEEE Transactions on Power Delivery, vol. 26, no. 1, pp. 378-384, 2011.

[12] Y. Zhu, T. Liu, C. Li, and Y. Liu, "Fast probability estimation of HVDC successive commutation failure caused by AC grid cascading failures," International Journal of Electrical Power \& Energy Systems, vol. 135, Article ID 107618, 2022.

[13] C. Wang, C. Zhang, X. Kong, P. Li, and Y. Yuan, "Procedure analysis of UHVDC commutation failure," The Journal of Engineering, vol. 2019, no. 16, pp. 3132-3134, 2019.

[14] F. R. V. D. A. Pedroso, M. T. Bassini, M. A. B. Horita, J. A. Jardini, J. F. Graham, and G. Liu, "HVDC multi-infeed analysis of the Brazilian transmission system and possible mitigation methods," CSEE Journal of Power and Energy Systems, vol. 4, no. 4, pp. 487-494, 2018.

[15] Y. Shao and Y. Tang, "Fast evaluation of commutation failure risk in multi-infeed HVDC systems," IEEE Transactions on Power Systems, vol. 33, no. 1, pp. 646-653, 2018.

[16] B. Rehman, C. Liu, W. Wei, C. Fu, and H. Li, "Applications of eigenvalues in installation of multi-infeed HVDC system for voltage stability," International Transactions on Electrical Energy Systems, vol. 30, Article ID e12645, 2020.

[17] H. Xiao, Y. Zhang, X. Duan, and Y. Li, "Evaluating strength of hybrid multi-infeed HVDC systems for planning studies using hybrid multi-infeed interactive effective short-circuit ratio," IEEE Transactions on Power Delivery, vol. 36, no. 4, pp. 2129-2144, 2021.

[18] H. Xiao, Y. Li, A. M. Gole, and X. Duan, "Computationally efficient and accurate approach for commutation failure risk areas identification in multi-infeed LCC-HVDC systems," IEEE Transactions on Power Electronics, vol. 35, no. 5, pp. 5238-5253, 2020. 
[19] T. Li, T. Zhao, M. Lv, L. Zou, and L. Zhang, "The mechanism and solution of the anomalous commutation failure of multiinfeed HVDC transmission systems," International Journal of Electrical Power \& Energy Systems, vol. 114, Article ID 105400, 2020.

[20] J. Ouyang, J. Ye, Z. Zhang, and Y. Diao, "Prevention control method of subsequent commutation failure for LCC-HVDC under grid fault," International Transactions on Electrical Energy Systems, vol. 31, no. 9, Article ID e13021, 2021.

[21] Y. Zhou, H. Wu, W. Wei, Y. Song, and H. Deng, "Optimal allocation of dynamic var sources for reducing the probability of commutation failure occurrence in the receiving-end systems," IEEE Transactions on Power Delivery, vol. 34, no. 1, pp. 324-333, 2019.

[22] B. Hu, T. Niu, F. Li, K. Xie, W. Li, and H. Jin, "Dynamic var reserve assessment in multi-infeed LCC-HVDC networks," IEEE Transactions on Power Systems, vol. 36, no. 1, pp. 68-80, 2021.

[23] H. Zhou, W. Yao, X. Ai, J. Zhang, J. Wen, and C. Li, "Coordinated power control of electrochemical energy storage for mitigating subsequent commutation failures of HVDC," International Journal of Electrical Power \& Energy Systems, vol. 134, Article ID 107455, 2022.

[24] C. Yin and F. Li, "Reactive power control strategy for inhibiting transient overvoltage caused by commutation failure," IEEE Transactions on Power Systems, vol. 36, no. 5, pp. 4764-4777, 2021.

[25] C. Guo, B. Liu, and C. Zhao, "Improved coordinated control approach for evolved CCC-HVDC system to enhance mitigation effect of commutation failure," Journal of Modern Power Systems and Clean Energy, vol. 9, no. 2, pp. 338-346, 2021.

[26] C. Zheng, Y. Tang, W. Zhang, C. Huang, and W. Qi, “Current order-based emergency control strategy for subsequent commutation failure elimination in HVDC," International Transactions on Electrical Energy Systems, vol. 31, no. 9, Article ID e13026, 2021.

[27] S. Mirsaeidi, D. Tzelepis, J. He, X. Dong, D. M. Said, and C. Booth, "A controllable thyristor-based commutation failure inhibitor for LCC-HVDC transmission systems," IEEE Transactions on Power Electronics, vol. 36, no. 4, pp. 37813792, 2021.

[28] L. Wang, W. Yao, Y. Xiong, Z. Shi, and J. Wen, "Commutation failure analysis and prevention of UHVDC system with hierarchical connection considering voltage harmonics," IEEE Transactions on Power Delivery, 2021, in press.

[29] L. Liu, S. Lin, K. Liao et al., "Extinction angle predictive control strategy for commutation failure mitigation in HVDC systems considering voltage distortion," IET Generation, Transmission \& Distribution, vol. 13, no. 22, pp. 5171-5179, 2019.

[30] W. Yao, C. Liu, J. Fang, X. Ai, J. Wen, and S. Cheng, "Probabilistic analysis of commutation failure in LCC-HVDC system considering the CFPREV and the initial fault voltage angle," IEEE Transactions on Power Delivery, vol. 35, no. 2, pp. $715-724,2020$.

[31] S. Mirsaeidi and X. Dong, "An enhanced strategy to inhibit commutation failure in line-commutated converters," IEEE Transactions on Industrial Electronics, vol. 67, no. 1, pp. 340-349, 2020.

[32] C. Petino, M. Heidemann, D. Eichhoff, M. Stumpe, E. Spahic, and F. Schettler, "Application of multilevel full bridge converters in HVDC multiterminal systems," IET Power Electronics, vol. 9, no. 2, pp. 297-304, 2016. 